\title{
Regenerable Thiophenolic Radical-Trapping Antioxidants
}

\author{
Jiajie Yan, ${ }^{\dagger}$ Jia-fei Poon,${ }^{\dagger}$ Vijay P. Singh, ${ }^{\dagger}$ Paul Gates, ${ }^{\dagger}$ and Lars Engman $*,{ }^{\dagger}$ \\ ${ }^{\dagger}$ Uppsala University, Department of Chemistry - BMC, Box 576, SE-751 23 Uppsala, Sweden \\ "University of Bristol, School of Chemistry, Bristol, BS8 1TS, United Kingdom.
}

\section{Supporting Information}

p S2-S11 Experimental Section

p S12 Table S1. Inhibited Rates of Conjugated Diene Formation $\left(R_{\text {inh }}\right)$ and Inhibition Times $\left(T_{\text {inh }}\right)$ with Acidified DTT $(0.5 \mathrm{mM})$

p S13 Table S2. Inhibited Rates of Conjugated Diene Formation $\left(R_{\text {inh }}\right)$ and Inhibition Times $\left(T_{\text {inh }}\right)$ with TCEP at Gradient Concentrations

p S14-S36 $\quad{ }^{1} \mathrm{H},{ }^{13} \mathrm{C},{ }^{77} \mathrm{Se}$, and ${ }^{125} \mathrm{Te}$ NMR Spectra for Compounds Prepared 


\section{Experimental Section}

\section{General}

${ }^{1} \mathrm{H}$ and ${ }^{13} \mathrm{C}$ NMR spectra were recorded on a $400 \mathrm{MHz}\left({ }^{1} \mathrm{H}: 399.97 \mathrm{MHz} ;{ }^{13} \mathrm{C} 100.57 \mathrm{MHz}\right)$ spectrometer, using the residual solvent peaks of $\mathrm{CDCl}_{3}\left({ }^{1} \mathrm{H}: \delta 7.26 ;{ }^{13} \mathrm{C}: \delta 77.0\right)$ as an indirect reference to TMS. ${ }^{77} \mathrm{Se}$ and ${ }^{125} \mathrm{Te}$ NMR were recorded on a $400 \mathrm{MHz}\left({ }^{77} \mathrm{Se}: 76.28\right.$ MHz; ${ }^{125}$ Te: $126.18 \mathrm{MHz}$ ) spectrometer using $\mathrm{Ph}_{2} \mathrm{Se}_{2}$ (460 ppm) and $\mathrm{Ph}_{2} \mathrm{Te}_{2}$ (423 ppm), respectively, as external standards. Melting points are uncorrected. Flash column chromatography was performed using silica gel $(0.04-0.06 \mathrm{~mm})$ or neutral aluminium oxide (50-200 $\mu \mathrm{m})$. Tetrahydrofuran was dried in a solvent purification system by passing it through an activated alumina column. Tetramethylethylenediamine (TMEDA), acetone, and hexane were dried over molecular sieves (4 $\AA$ ) for 2 days. Dibutyl ditelluride ${ }^{1}$, dioctyl ditelluride $^{2}$, and dihexadecyl ditelluride ${ }^{3}$ were prepared according to the literature.

Dioctyl diselenide. Sodium chips (104 mg, $4.5 \mathrm{mmol}$ ), selenium powder (356 mg, $4.5 \mathrm{mmol}$ ), and naphthalene $(69 \mathrm{mg}, 0.54 \mathrm{mmol})$ were mixed together in degassed dry THF $(20 \mathrm{~mL})$ under nitrogen at room temperature. The resulting mixture was stirred overnight to afford a brown suspension. 1-Bromooctane $(0.4 \mathrm{~mL}, 2.3 \mathrm{mmol})$ was added to the suspension and stirred for an additional $4 \mathrm{~h}$ at room temperature. The reaction was then quenched with water and extracted with diethyl ether. The organic phase was washed with brine, dried over magnesium sulfate, filtered, and evaporated under reduced pressure. The residue was purified by flash column chromatography on silica gel (pentane) to afford the title compound as a yellow oil (258 mg, 58\%). ${ }^{1} \mathrm{H}$ NMR (400 MHz, $\left.\mathrm{CDCl}_{3}\right): \delta 2.91(\mathrm{t}, J=7.6 \mathrm{~Hz}, 4 \mathrm{H}), 1.72(\mathrm{~m}$, 4H), 1.28-1.40 (several peaks, 20H), $0.88(\mathrm{t}, J=7.2 \mathrm{~Hz}, 6 \mathrm{H}) .{ }^{13} \mathrm{C} \mathrm{NMR}\left(100 \mathrm{MHz}, \mathrm{CDCl}_{3}\right): \delta$ 
31.8, 31.0, 30.3, 29.5, 29.2, 29.1, 22.6, 14.1. ${ }^{77} \mathrm{Se}$ NMR $\left(76 \mathrm{MHz}, \mathrm{CDCl}_{3}\right): \delta 307 .{ }^{1} \mathrm{H} \mathrm{NMR}$ data were in reasonable agreement with those reported in the literature. ${ }^{4}$

Bis-2-(butyltelluro)phenyl disulfide (6a). $n$-BuLi (1.6 $\mathrm{M}$ in hexanes, $2.8 \mathrm{~mL}, 4.4 \mathrm{mmol}$ ) was added dropwise to a solution of thiophenol $(0.21 \mathrm{~mL}, 2.0 \mathrm{mmol})$ in anhydrous TMEDA $(1.0 \mathrm{~mL})$ under nitrogen at $-78{ }^{\circ} \mathrm{C}$. The resulting solid was then allowed to warm to room temperature and the solution stirred overnight to give a pale yellow suspension. ${ }^{5}$ Dibutyl ditelluride $(812 \mathrm{mg}, 2.2 \mathrm{mmol})$ was then added. After stirring at room temperature for $4 \mathrm{~h}$, the reaction was opened to the atmosphere, quenched with saturated ammonium chloride (20 $\mathrm{mL})$, and extracted with diethyl ether $(20 \mathrm{~mL} \times 3)$. The organic phase was washed with brine, dried over magnesium sulfate, filtered, and evaporated under reduced pressure. The residue was purified by flash column chromatography on neutral aluminium oxide (pentane to pentane $:$ ethyl acetate $=50: 1)$ to afford the title compound as a yellow oil $(252 \mathrm{mg}, 43 \%)$. ${ }^{1} \mathrm{H}$ NMR $\left(400 \mathrm{MHz}, \mathrm{CDCl}_{3}\right): \delta 7.65(\mathrm{dd}, J=1.6,7.6 \mathrm{~Hz}, 2 \mathrm{H}), 7.52(\mathrm{dd}, J=1.6,7.6 \mathrm{~Hz}, 2 \mathrm{H})$, $7.21(\mathrm{ddd}, J=1.6,7.6,7.6 \mathrm{~Hz}, 2 \mathrm{H}), 7.04(\mathrm{ddd}, J=1.6,7.6,7.6 \mathrm{~Hz}, 2 \mathrm{H}), 2.93(\mathrm{t}, J=7.6 \mathrm{~Hz}$, $4 \mathrm{H}), 1.80(\mathrm{~m}, 4 \mathrm{H}), 1.42(\mathrm{~m}, 4 \mathrm{H}), 0.92(\mathrm{t}, J=7.6 \mathrm{~Hz}, 6 \mathrm{H}) .{ }^{13} \mathrm{C} \mathrm{NMR}\left(100 \mathrm{MHz}, \mathrm{CDCl}_{3}\right): \delta$ 142.4, 138.4, 128.7, 127.5, 127.2, 114.5, 33.4, 25.1, 13.4, 9.6. ${ }^{125}$ Te NMR (126 MHz, $\left.\mathrm{CDCl}_{3}\right)$ : $\delta$ 427. HRMS (TOF MS EI ${ }^{+}$) m/z: [M] $]^{+}$calcd for $\mathrm{C}_{20} \mathrm{H}_{26} \mathrm{~S}_{2} \mathrm{Te}_{2}$ 589.9601; found 589.9606.

Bis-2-(octyltelluro)phenyl disulfide (6b). Thiophenol (0.21 mL, $2.0 \mathrm{mmol}), n$-BuLi (1.6 M in hexanes, $2.8 \mathrm{~mL}, 4.4 \mathrm{mmol})$, TMEDA (1.0 mL), and dioctyl ditelluride (1.06 g, $2.2 \mathrm{mmol}$ ) were reacted following the procedure described for $\mathbf{6 a}$ to afford the title compound as an orange oil (227mg, 33\%). ${ }^{1} \mathrm{H}$ NMR (400 MHz, $\left.\mathrm{CDCl}_{3}\right): \delta 7.65(\mathrm{dd}, J=1.2,7.6 \mathrm{~Hz}, 2 \mathrm{H}), 7.52$ $(\mathrm{dd}, J=1.2,7.6 \mathrm{~Hz}, 2 \mathrm{H}), 7.21(\mathrm{ddd}, J=1.2,7.6,7.6 \mathrm{~Hz}, 2 \mathrm{H}), 7.04(\mathrm{ddd}, J=1.2,7.6,7.6 \mathrm{~Hz}$, 
2H), $2.92(\mathrm{t}, J=7.6 \mathrm{~Hz}, 4 \mathrm{H}), 1.81(\mathrm{~m}, 4 \mathrm{H}), 1.26-1.43$ (several peaks, $20 \mathrm{H}), 0.88(\mathrm{t}, J=7.2$ $\mathrm{Hz}, 6 \mathrm{H}) .{ }^{13} \mathrm{C} \mathrm{NMR}\left(100 \mathrm{MHz}, \mathrm{CDCl}_{3}\right): \delta 142.5,138.4,128.7,127.4,127.2,114.5,32.0,31.8$, 31.3, 29.2, 28.9, 22.6, 14.1, 10.0. ${ }^{125} \mathrm{Te}$ NMR (126 MHz, $\left.\mathrm{CDCl}_{3}\right): \delta$ 428. HRMS (TOF MS $\mathrm{EI}^{+}$) $m / z:[\mathrm{M}]^{+}$calcd for $\mathrm{C}_{28} \mathrm{H}_{42} \mathrm{~S}_{2} \mathrm{Te}_{2}$ 702.0853; found 702.0840.

Bis-2-(hexadecyltelluro)phenyl disulfide (6c). Thiophenol (0.21 mL, $2.0 \mathrm{mmol}), n$-BuLi (1.6 M in hexanes, $2.8 \mathrm{~mL}, 4.4 \mathrm{mmol})$, TMEDA $(1.0 \mathrm{~mL})$, and dihexadecyl ditelluride $(1.55 \mathrm{~g}$, $2.2 \mathrm{mmol}$ ) were reacted following the procedure described for $\mathbf{6 a}$ to afford the title compound as a yellow solid $(177 \mathrm{mg}, 19 \%)$ mp: $48-51{ }^{\circ} \mathrm{C} .{ }^{1} \mathrm{H}$ NMR $\left(400 \mathrm{MHz}, \mathrm{CDCl}_{3}\right): \delta 7.64(\mathrm{dd}, J=$ 1.2, 7.6 Hz, 2H), $7.52(\mathrm{dd}, J=1.2,7.6 \mathrm{~Hz}, 2 \mathrm{H}), 7.21(\mathrm{ddd}, J=1.2,7.6,7.6 \mathrm{~Hz}, 2 \mathrm{H}), 7.03$ $(\mathrm{ddd}, J=1.2,7.6,7.6 \mathrm{~Hz}, 2 \mathrm{H}), 2.92(\mathrm{t}, J=7.6 \mathrm{~Hz}, 4 \mathrm{H}), 1.81(\mathrm{~m}, 4 \mathrm{H}), 1.26-1.43$ (several peaks, $52 \mathrm{H}), 0.88(\mathrm{t}, J=7.2 \mathrm{~Hz}, 6 \mathrm{H}) .{ }^{13} \mathrm{C} \mathrm{NMR}\left(100 \mathrm{MHz}, \mathrm{CDCl}_{3}\right): \delta 142.5,138.4,128.7$, $127.4,127.2,114.5,32.0,31.9,31.3,29.7$ (3C), 29.6, 29.5, 29.4, 29.0, 22.7, 14.1, 10.0. ${ }^{125} \mathrm{Te}$ NMR (126 MHz, $\mathrm{CDCl}_{3}$ ): $\delta$ 428. HRMS (TOF MS EI $\left.{ }^{+}\right) m / z:[\mathrm{M}]^{+}$calcd for $\mathrm{C}_{44} \mathrm{H}_{74} \mathrm{~S}_{2} \mathrm{Te}_{2}$ 926.3357; found 926.3340.

Bis-2-(octylseleno)phenyl disulfide (6d). Thiophenol (0.21 mL, $2.0 \mathrm{mmol}), n$-BuLi $(1.6 \mathrm{M}$ in hexanes, $2.8 \mathrm{~mL}, 4.4 \mathrm{mmol})$, TMEDA $(1.0 \mathrm{~mL})$, and dioctyl diselenide ( $845 \mathrm{mg}, 2.2 \mathrm{mmol})$ were reacted following the procedure described for $\mathbf{6 a}$ to afford the title compound as a pale yellow oil (161 mg, 27\%). ${ }^{1} \mathrm{H}$ NMR (400 MHz, $\left.\mathrm{CDCl}_{3}\right): \delta 7.51(\mathrm{~m}, 4 \mathrm{H}), 7.20(\mathrm{~m}, 2 \mathrm{H}), 7.10$ (m, 2H), $2.95(\mathrm{t}, J=7.6 \mathrm{~Hz}, 4 \mathrm{H}), 1.72(\mathrm{~m}, 4 \mathrm{H}), 1.27-1.44$ (several peaks, $20 \mathrm{H}), 0.88(\mathrm{t}, J=$ $7.2 \mathrm{~Hz}, 6 \mathrm{H}) .{ }^{13} \mathrm{C} \mathrm{NMR}\left(100 \mathrm{MHz}, \mathrm{CDCl}_{3}\right): \delta 140.4,134.7,128.7,128.4,126.8,126.2,31.8$, 30.0, 29.8, 29.2 (2C), 29.1, 22.6, 14.1. ${ }^{77} \mathrm{Se}$ NMR (76 MHz, $\mathrm{CDCl}_{3}$ ): $\delta$ 255. HRMS (TOF MS $\mathrm{EI}^{+}$) $m / z:[\mathrm{M}]^{+}$calcd for $\mathrm{C}_{28} \mathrm{H}_{42} \mathrm{~S}_{2} \mathrm{Se}_{2}$ 602.1058; found 602.1041 . 
Bis-4-(butyltelluro)phenyl disulfide (7a). $t$ - $\mathrm{BuLi}(1.7 \mathrm{M}$ in pentane, $3.5 \mathrm{~mL}, 6.0 \mathrm{mmol})$ was added dropwise to a solution of 4-bromothiophenol (378 mg, $2.0 \mathrm{mmol})$ in dry THF (15 mL) under nitrogen at $-78{ }^{\circ} \mathrm{C}$. After stirring of the resulting yellow solution for $2 \mathrm{~h}$ at $-78{ }^{\circ} \mathrm{C}$, dibutyl ditelluride $(812 \mathrm{mg}, 2.2 \mathrm{mmol}$ ) was added. The mixture was then allowed to warm to room temperature and stirred for an additional $4 \mathrm{~h}$. The flask was then opened to the atmosphere and a saturated ammonium chloride solution was added before extraction with diethyl ether $(20 \mathrm{~mL} \times 3)$. The organic phase was washed with brine, dried over magnesium sulfate, filtered, and evaporated under reduced pressure. The residue was purified by flash column chromatography on silica gel (pentane) to afford the title compound as an orange oil (185 mg, 32\%). ${ }^{1} \mathrm{H}$ NMR (400 MHz, $\left.\mathrm{CDCl}_{3}\right): \delta 7.62(\mathrm{~d}, J=8.0 \mathrm{~Hz}, 4 \mathrm{H}), 7.30(\mathrm{~d}, J=8.0 \mathrm{~Hz}$, 4H), $2.89(\mathrm{t}, J=7.6 \mathrm{~Hz}, 4 \mathrm{H}), 1.77(\mathrm{~m}, 4 \mathrm{H}), 1.39(\mathrm{~m}, 4 \mathrm{H}), 0.90(\mathrm{t}, J=7.6 \mathrm{~Hz}, 6 \mathrm{H}) .{ }^{13} \mathrm{C} \mathrm{NMR}$ $\left(100 \mathrm{MHz}, \mathrm{CDCl}_{3}\right): \delta 138.6,136.5,128.1,110.7,33.8,25.0,13.4,8.8 .{ }^{125} \mathrm{Te} \mathrm{NMR}(126 \mathrm{MHz}$, $\mathrm{CDCl}_{3}$ ): $\delta$ 512. HRMS (TOF MS EI ${ }^{+}$) m/z: $[\mathrm{M}]^{+}$calcd for $\mathrm{C}_{20} \mathrm{H}_{26} \mathrm{~S}_{2} \mathrm{Te}_{2}$ 589.9601; found 589.9601.

Bis-4-(octyltelluro)phenyl disulfide (7b). 4-Bromothiophenol (378 mg, $2.0 \mathrm{mmol}), t$-BuLi (1.7 $\mathrm{M}$ in pentane, $3.5 \mathrm{~mL}, 6.0 \mathrm{mmol})$, and dioctyl ditelluride (1.06 g, $2.2 \mathrm{mmol}$ ) were reacted in dry THF (15 mL) following the procedure described for $\mathbf{7 a}$ to afford the title compound as an orange oil (189 mg, 27\%). ${ }^{1} \mathrm{H}$ NMR $\left(400 \mathrm{MHz} \mathrm{CDCl}_{3}\right): \delta 7.62(\mathrm{~d}, J=8.0 \mathrm{~Hz}, 4 \mathrm{H}), 7.28$ (d, $J=8.0 \mathrm{~Hz}, 4 \mathrm{H}), 2.88(\mathrm{t}, J=7.6 \mathrm{~Hz}, 4 \mathrm{H}), 1.78(\mathrm{~m}, 4 \mathrm{H}), 1.25-1.37$ (several peaks, 20H), $0.87(\mathrm{t}, J=7.2 \mathrm{~Hz}, 6 \mathrm{H}) .{ }^{13} \mathrm{C} \mathrm{NMR}\left(100 \mathrm{MHz}, \mathrm{CDCl}_{3}\right): \delta 138.6,136.5,128.1,110.8,31.9$, 31.8, 31.7, 29.1, 28.8, 22.6, 14.1, 9.1. ${ }^{125} \mathrm{Te}$ NMR (126 MHz, $\left.\mathrm{CDCl}_{3}\right): \delta 512 . \mathrm{HRMS}$ (TOF MS EI $\left.{ }^{+}\right) m / z:[\mathrm{M}]^{+}$calcd for $\mathrm{C}_{28} \mathrm{H}_{42} \mathrm{~S}_{2} \mathrm{Te}_{2}$ 702.0853; found 702.0870. 
Bis-4-(hexadecyltelluro)phenyl disulfide (7c). 4-Bromothiophenol (378 mg, $2.0 \mathrm{mmol}), t$ BuLi (1.7 $\mathrm{M}$ in pentane, $3.5 \mathrm{~mL}, 6.0 \mathrm{mmol})$, and dihexadecyl ditelluride (1.55 g, $2.2 \mathrm{mmol})$ were reacted in dry THF $(15 \mathrm{~mL})$ following the procedure described for $7 \mathbf{a}$ to afford the title compound as a pale yellow solid (139 mg, 15\%). mp: 59-62 ${ }^{\circ} \mathrm{C} .{ }^{1} \mathrm{H} \mathrm{NMR}\left(400 \mathrm{MHz}, \mathrm{CDCl}_{3}\right)$ : $\delta 7.61(\mathrm{~d}, J=8.0 \mathrm{~Hz}, 4 \mathrm{H}), 7.30(\mathrm{~d}, J=8.0 \mathrm{~Hz}, 4 \mathrm{H}), 2.88(\mathrm{t}, J=7.6 \mathrm{~Hz}, 4 \mathrm{H}), 1.78(\mathrm{~m}, 4 \mathrm{H})$, 1.24-1.37 (several peaks, 52H), $0.88(\mathrm{t}, J=7.2 \mathrm{~Hz}, 6 \mathrm{H}) \cdot{ }^{13} \mathrm{C} \mathrm{NMR}\left(100 \mathrm{MHz}, \mathrm{CDCl}_{3}\right): \delta$ 138.6, 136.5, 128.1, 110.8, 31.9 (2C), 31.7, 29.7 (2C), 29.6 (2C), 29.5, 29.4, 28.9, 22.7, 14.1, 9.1. ${ }^{125}$ Te NMR (126 MHz, $\mathrm{CDCl}_{3}$ ): $\delta$ 512. HRMS (TOF MS EI ${ }^{+}$) $m / z:[\mathrm{M}]^{+}$calcd for $\mathrm{C}_{44} \mathrm{H}_{74}$ $\mathrm{S}_{2} \mathrm{Te}_{2}$ 926.3357; found 926.3331.

4-(Octylseleno)thiophenol (8). 4-Bromothiophenol (227 mg, $1.2 \mathrm{mmol}), t$-BuLi (1.7 $\mathrm{M}$ in pentane, $2.1 \mathrm{~mL}, 3.6 \mathrm{mmol})$ and dioctyl diselenide $(461 \mathrm{mg}, 1.2 \mathrm{mmol})$ were reacted in dry THF (10 $\mathrm{mL}$ ) following the procedure described for $\mathbf{7 a}$ to afford the title compound as a yellow oil (141 mg, 39\%). ${ }^{1} \mathrm{H} \mathrm{NMR}\left(400 \mathrm{MHz}, \mathrm{CDCl}_{3}\right): \delta 7.35(\mathrm{~d}, J=8.4 \mathrm{~Hz}, 2 \mathrm{H}), 7.16(\mathrm{~d}, J$ $=8.4 \mathrm{~Hz}, 2 \mathrm{H}), 3.42(\mathrm{~s}, 1 \mathrm{H}), 2.86(\mathrm{t}, J=7.6 \mathrm{~Hz}, 2 \mathrm{H}), 1.67(\mathrm{~m}, 2 \mathrm{H}), 1.26-1.40$ (several peaks, $10 \mathrm{H}), 0.88(\mathrm{t}, J=6.8 \mathrm{~Hz}, 3 \mathrm{H}) .{ }^{13} \mathrm{C} \mathrm{NMR}\left(100 \mathrm{MHz}, \mathrm{CDCl}_{3}\right): \delta 133.4,130.0,129.2,127.7$, 31.8, 30.1, 29.8, 29.1, 29.0, 28.3, 22.6, 14.1. ${ }^{77} \mathrm{Se}$ NMR (76 MHz, $\left.\mathrm{CDCl}_{3}\right): \delta 290 . \mathrm{HRMS}$ (TOF MS EI ${ }^{+}$) $m / z:[\mathrm{M}]^{+}$calcd for $\mathrm{C}_{14} \mathrm{H}_{22} \mathrm{SSe}$ 302.0607; found 302.0613.

Bis-4-(octylseleno)phenyl disulfide (7d). ${ }^{6}$ A solution of potassium ferricyanide $(112 \mathrm{mg}$, $0.34 \mathrm{mmol})$ in water $(2 \mathrm{~mL})$ was added to a stirring mixture of compound $\mathbf{8}(104 \mathrm{mg}, 0.34$ $\mathrm{mmol})$ and $\mathrm{NaOH}(300 \mathrm{mg}, 7.5 \mathrm{mmol})$ in water $(3 \mathrm{~mL})$. After stirring for overnight, water (20 $\mathrm{mL}$ ) was added and the solution was extracted with ethyl acetate $(20 \mathrm{~mL} \times 3)$. The organic layer was washed with brine, dried over magnesium sulfate, filtered and evaporated under reduced pressure. The residue was purified by flash column chromatography on silica gel 
(pentane) to afford the title compound as a pale yellow solid (102 mg, 100\%). mp: $42-44{ }^{\circ} \mathrm{C}$. ${ }^{1} \mathrm{H}$ NMR $\left(400 \mathrm{MHz}, \mathrm{CDCl}_{3}\right): \delta 7.39(\mathrm{~d}, J=8.8 \mathrm{~Hz}, 4 \mathrm{H}), 7.36(\mathrm{~d}, J=8.8 \mathrm{~Hz}, 4 \mathrm{H}), 2.89(\mathrm{t}, J=$ $7.2 \mathrm{~Hz}, 4 \mathrm{H}), 1.69(\mathrm{~m}, 4 \mathrm{H}), 1.26-1.41$ (several peaks, $20 \mathrm{H}), 0.88(\mathrm{t}, J=6.4 \mathrm{~Hz}, 6 \mathrm{H}) .{ }^{13} \mathrm{C} \mathrm{NMR}$ $\left(100 \mathrm{MHz}, \mathrm{CDCl}_{3}\right): \delta 135.2,132.6,130.4,128.5,31.8,30.0,29.8,29.1,29.0,28.0,22.6,14.1$. ${ }^{77} \mathrm{Se} \mathrm{NMR}\left(76 \mathrm{MHz}, \mathrm{CDCl}_{3}\right): \delta 292$. HRMS (TOF MS EI $\left.{ }^{+}\right) \mathrm{m} / z:[\mathrm{M}]^{+}$calcd for $\mathrm{C}_{28} \mathrm{H}_{42} \mathrm{~S}_{2} \mathrm{Se}_{2}$ 602.1058; found 602.1080.

Bis-3-(octyltelluro)phenyl disulfide (9a). 3-Bromothiophenol (378 mg, $2.0 \mathrm{mmol}$ ), $t$-BuLi (1.7 $\mathrm{M}$ in pentane, $3.5 \mathrm{~mL}, 6.0 \mathrm{mmol})$, and dioctyl ditelluride (1.06 g, $2.2 \mathrm{mmol})$ were reacted in dry THF (15 mL) following the procedure described for $\mathbf{7 a}$ to afford the title compound as a yellow oil (243 mg, 35\%). ${ }^{1} \mathrm{H}$ NMR (400 MHz, $\left.\mathrm{CDCl}_{3}\right): \delta 7.79(\mathrm{~s}, 2 \mathrm{H}), 7.54(\mathrm{~d}, J=7.6 \mathrm{~Hz}$, 2H), $7.36(\mathrm{~d}, J=7.6 \mathrm{~Hz}, 2 \mathrm{H}), 7.11(\mathrm{t}, J=7.6 \mathrm{~Hz}, 2 \mathrm{H}), 2.88(\mathrm{t}, J=7.6 \mathrm{~Hz}, 4 \mathrm{H}), 1.76(\mathrm{~m}, 4 \mathrm{H})$, 1.25-1.35 (several peaks, 20H), $0.88(\mathrm{t}, J=7.2 \mathrm{~Hz}, 6 \mathrm{H}) \cdot{ }^{13} \mathrm{C} \mathrm{NMR}\left(100 \mathrm{MHz}, \mathrm{CDCl}_{3}\right): \delta$ $137.6,136.5,136.1,129.5,126.4,112.9,31.9,31.8,31.7,29.2,28.9,22.6,14.1,9.2 .{ }^{125} \mathrm{Te}$ NMR (126 MHz, $\mathrm{CDCl}_{3}$ ): $\delta$ 530. HRMS (TOF MS EI ${ }^{+}$) m/z: [M] $]^{+}$calcd for $\mathrm{C}_{28} \mathrm{H}_{42} \mathrm{~S}_{2} \mathrm{Te}_{2}$ 702.0853; found 702.0862.

3-(Octylseleno)thiophenol. 3-Bromothiophenol (378 mg, $2.0 \mathrm{mmol}), t$-BuLi (1.7 $\mathrm{M}$ in pentane, $3.5 \mathrm{~mL}, 6.0 \mathrm{mmol})$ and dioctyl diselenide $(845 \mathrm{mg}, 2.2 \mathrm{mmol})$ were reacted in dry THF (15 mL) following the procedure described for $7 \mathbf{a}$ to afford the title compound as a pale yellow oil (364 mg, 60\%). ${ }^{1} \mathrm{H}$ NMR (400 MHz, $\left.\mathrm{CDCl}_{3}\right): \delta 7.39(\mathrm{~s}, 1 \mathrm{H}), 7.24(\mathrm{~m}, 1 \mathrm{H}), 7.11(\mathrm{~m}$, 2H), $3.44(\mathrm{~s}, 1 \mathrm{H}), 2.90(\mathrm{t}, J=7.6 \mathrm{~Hz}, 2 \mathrm{H}), 1.70(\mathrm{~m}, 2 \mathrm{H}), 1.26-1.43$ (several peaks, $10 \mathrm{H}), 0.88$ $(\mathrm{t}, J=7.2 \mathrm{~Hz}, 3 \mathrm{H}) .{ }^{13} \mathrm{C} \mathrm{NMR}\left(100 \mathrm{MHz}, \mathrm{CDCl}_{3}\right): \delta 132.5,131.8,131.6,129.4,129.3,127.4$, 31.8, 30.0, 29.8, 29.1, 29.0, 27.9, 22.6, 14.1. ${ }^{77} \mathrm{Se} \mathrm{NMR}\left(76 \mathrm{MHz}, \mathrm{CDCl}_{3}\right): \delta 298 . \mathrm{HRMS}$ (TOF MS EI ${ }^{+}$) m/z: $[\mathrm{M}]^{+}$calcd for $\mathrm{C}_{14} \mathrm{H}_{22} \mathrm{SSe}$ 302.0607; found 302.0606. 
Bis-3-(octylseleno)phenyl disulfide (9b). 3-(Octylseleno)thiophenol (205 mg, 0.68 mmol), $\mathrm{NaOH}$ (408 mg, $10.2 \mathrm{mmol}$ ) and potassium ferricyanide (224 mg, $0.68 \mathrm{mmol}$ ) were reacted in water $(6 \mathrm{~mL})$ following the procedure described for $\mathbf{7 d}$ to afford the title compound as a pale yellow oil (200 mg, 98\%). ${ }^{1} \mathrm{H}$ NMR (400 MHz, $\left.\mathrm{CDCl}_{3}\right): \delta 7.57(\mathrm{~s}, 2 \mathrm{H}), 7.31$ (d, J = $7.6 \mathrm{~Hz}$, 4H), $7.17(\mathrm{t}, J=7.6 \mathrm{~Hz}, 2 \mathrm{H}), 2.87(\mathrm{t}, J=7.6 \mathrm{~Hz}, 4 \mathrm{H}), 1.67(\mathrm{~m}, 4 \mathrm{H}), 1.26-1.38$ (several peaks, 20H), $0.88(\mathrm{t}, J=7.2 \mathrm{~Hz}, 6 \mathrm{H}) .{ }^{13} \mathrm{C} \mathrm{NMR}\left(100 \mathrm{MHz}, \mathrm{CDCl}_{3}\right): \delta 137.6,132.2,130.6,130.1$, 129.4, 125.4, 31.8, 30.0, 29.8, 29.2, 29.0, 27.8, 22.6, 14.1. ${ }^{77} \mathrm{Se} \mathrm{NMR}\left(76 \mathrm{MHz}, \mathrm{CDCl}_{3}\right): \delta$ 300. HRMS (TOF MS EI ${ }^{+}$) m/z: [M] ${ }^{+}$calcd for $\mathrm{C}_{28} \mathrm{H}_{42} \mathrm{~S}_{2} \mathrm{Se}_{2}$ 602.1058; found 602.1060.

Isopropyl phenyl sulfide (10). A mixture of thiophenol $(0.31 \mathrm{~mL}, 3.0 \mathrm{mmol})$, anhydrous potassium carbonate $(580 \mathrm{mg}, 4.2 \mathrm{mmol})$, 2-bromopropane $(0.34 \mathrm{~mL}, 3.6 \mathrm{~mL})$ and dry acetone $(10 \mathrm{~mL})$ were mixed under nitrogen and heated at reflux overnight. After completion, the reaction mixture was cooled to room temperature, quenched with water $(30 \mathrm{~mL})$, and extracted with diethyl ether $(30 \mathrm{~mL} \times 3)$. The organic phase was then washed with brine, dried over magnesium sulfate, filtered, and evaporated under reduced pressure. The residue was purified by flash column chromatography on silica gel (pentane) to afford the title compound as a colorless oil (389 mg, 87\%). ${ }^{1} \mathrm{H}$ and ${ }^{13} \mathrm{C}$ NMR data were in good agreement with those reported in the literature. ${ }^{7}$

Isopropyl 2-(octyltelluro)phenyl sulfide (11). $n$-BuLi (1.6 M in hexanes, $0.69 \mathrm{~mL}, 1.1$ mmol) was added dropwise to a solution of compound $\mathbf{1 0}(152 \mathrm{mg}, 1.0 \mathrm{mmol})$ in dry hexane $(5 \mathrm{~mL})$ under nitrogen at $0{ }^{\circ} \mathrm{C}$. Anhydrous TMEDA $(0.17 \mathrm{~mL}, 1.1 \mathrm{mmol})$ was then added to the mixture within $10 \mathrm{~min}$. The reaction was allowed to warm to room temperature and stirred for $3 \mathrm{~h}^{8}{ }^{8}$ Tellurium powder $(140 \mathrm{mg}, 1.1 \mathrm{mmol})$ was then added. After stirring for $2 \mathrm{~h}$, 
the reaction was opened to air, quenched with water $(10 \mathrm{~mL})$ and extracted with diethyl ether $(10 \mathrm{~mL} \times 3)$. The organic phase was washed with brine, dried over magnesium sulfate, filtered, and evaporated under reduced pressure to obtain crude bis-2-(isopropylthio)phenyl ditelluride as a red oil. The crude product was dissolved in anhydrous ethanol (10 mL) under nitrogen at room temperature and sodium borohydride $(190 \mathrm{mg}, 5.0 \mathrm{mmol})$ was added. Then 1-bromooctane $(0.17 \mathrm{~mL}, 1.0 \mathrm{mmol})$ was syringed into the mixture and the reaction was left for overnight. After the addition of water $(10 \mathrm{~mL})$ and extraction with diethyl ether $(10 \mathrm{~mL} \times$ 3), the organic phase was washed with brine, dried over magnesium sulfate, filtered, and evaporated under reduced pressure. The residue was then purified by flash column chromatography on silica gel (pentane) to afford the title compound as a pale yellow oil (163 mg, 42\%). ${ }^{1} \mathrm{H}$ NMR (400 MHz, $\left.\mathrm{CDCl}_{3}\right): \delta 7.37(\mathrm{~m}, 2 \mathrm{H}), 7.14(\mathrm{~m}, 2 \mathrm{H}), 3.35(\mathrm{~m}, 1 \mathrm{H}), 2.81(\mathrm{t}$, $J=7.6 \mathrm{~Hz}, 2 \mathrm{H}), 1.85(\mathrm{~m}, 2 \mathrm{H}), 1.28-1.47$ (several peaks, $16 \mathrm{H}), 0.88(\mathrm{t}, J=7.2 \mathrm{~Hz}, 3 \mathrm{H}) .{ }^{13} \mathrm{C}$ NMR (100 MHz, $\left.\mathrm{CDCl}_{3}\right): \delta 138.5,133.9,132.8,128.3,126.6,125.7,40.1,32.4,31.8,30.9$, 29.2, 29.0, 23.1, 22.6, 14.1, 8.1. ${ }^{125}$ Te NMR (126 MHz, $\left.\mathrm{CDCl}_{3}\right): \delta$ 434. HRMS (TOF MS $\mathrm{EI}^{+}$) $m / z:[\mathrm{M}]^{+}$calcd for $\mathrm{C}_{17} \mathrm{H}_{28} \mathrm{STe}$ 394.0974; found 394.0988.

4-Bromophenyl isopropyl sulfide (12). 4-Bromothiophenol (567 mg, $3.0 \mathrm{mmol}$ ), anhydrous potassium carbonate $(580 \mathrm{mg}, 4.2 \mathrm{mmol}), 2$-bromopropane $(0.34 \mathrm{~mL}, 3.6 \mathrm{~mL})$ were reacted in dry acetone $(10 \mathrm{~mL})$ following the procedure described for $\mathbf{1 0}$ to afford the title compound as a pale yellow oil $(663 \mathrm{mg}, 96 \%) .{ }^{1} \mathrm{H}$ and ${ }^{13} \mathrm{C}$ NMR data were in good agreement with those reported in the literature. ${ }^{9}$

Isopropyl 4-(octyltelluro)phenyl sulfide (13). $t$-BuLi (1.7 $\mathrm{M}$ in pentane, $1.2 \mathrm{~mL}, 2.0 \mathrm{mmol})$ was added dropwise to a solution of 4-bromophenyl isopropyl sulfide $(231 \mathrm{mg}, 1.0 \mathrm{mmol})$ in dry THF $(10 \mathrm{~mL})$ under nitrogen at $-78^{\circ} \mathrm{C}$ and the mixture was stirred for $2 \mathrm{~h}$, Tellurium 
powder (140 mg, $1.1 \mathrm{mmol})$ was added and the flask was allowed to warm to room temperature. After stirring overnight, air was introduced and the reaction was quenched with water $(10 \mathrm{~mL})$ and extracted with diethyl ether $(10 \mathrm{~mL} \times 3)$. The organic phase was washed with brine, dried over magnesium sulfate, filtered, and evaporated under reduced pressure to obtain crude bis-4-(isopropylthio)phenyl ditelluride as a red oil. The crude product, sodium borohydride $(190 \mathrm{mg}, 5.0 \mathrm{mmol})$, and 1-bromooctane $(0.17 \mathrm{~mL}, 1.0 \mathrm{mmol})$ were reacted in anhydrous ethanol $(10 \mathrm{~mL})$ following the procedure described for $\mathbf{1 1}$ to afford the title compound as a pale yellow oil $(171 \mathrm{mg}, 44 \%) .{ }^{1} \mathrm{H}$ NMR $\left(400 \mathrm{MHz}, \mathrm{CDCl}_{3}\right): \delta 7.61(\mathrm{~d}, J=$ $8.4 \mathrm{~Hz}, 2 \mathrm{H}), 7.20(\mathrm{~d}, J=8.4 \mathrm{~Hz}, 2 \mathrm{H}), 3.37(\mathrm{~m}, 1 \mathrm{H}), 2.89(\mathrm{t}, J=7.6 \mathrm{~Hz}, 2 \mathrm{H}), 1.79(\mathrm{~m}, 2 \mathrm{H})$, 1.25-1.37 (several peaks, $16 \mathrm{H}), 0.87(\mathrm{t}, J=7.2 \mathrm{~Hz}, 3 \mathrm{H}) \cdot{ }^{13} \mathrm{C} \mathrm{NMR}\left(100 \mathrm{MHz}, \mathrm{CDCl}_{3}\right): \delta$ 138.5, 135.3 132.0, 109.7, 38.0, 31.9, 31.8, 31.7, 29.1, 28.8, 23.1, 22.6, 14.1, 9.0. ${ }^{125}$ Te NMR $\left(126 \mathrm{MHz}, \mathrm{CDCl}_{3}\right.$ ): $\delta$ 507. HRMS (TOF MS EI ${ }^{+}$) m/z: [M] ${ }^{+}$calcd for $\mathrm{C}_{17} \mathrm{H}_{28} \mathrm{STe}$ 394.0974; found 394.0988 .

HPLC Peroxidation Assay. The experimental setup for recording inhibited rates of peroxidation $\left(R_{\text {inh }}\right)$ and inhibition times $\left(T_{\text {inh }}\right)$ in a water chlorobenzene two-phase system during azo-initiated peroxidation of linoleic acid has been recently described. ${ }^{10}$ Values reported in Table 1, S1, and S2 for reactions performed in the presence of different coantioxidants are means \pm SD triplicates.

\section{References}

(1) Engman, L.; Cava, M. P. Synth. Commun. 1982, 12, 163-165.

(2) Li, Y.; Silverton, L. C.; Haasch, R.; Tong, Y. Y. Langmuir, 2008, 24, 7048-7053. 
(3) Nakamura, T.; Miyamae, T.; Nakai, L.; Kondoh, H.; Kawamoto, T.; Kobayashi, N.; Yasuda, S.; Yoshimura, D.; Ohta, T.; Nozoye, H.; Matsumoto, M. Lagmuir, 2005, 21, 33443353.

(4) Higuchi, H.; Otsubo, T.; Ogura, F.; Yamaguchi, H.; Sakata, Y.; Misumi, S. Bull. Chem. Soc. Jpn. 1982, 55, 182-187.

(5) Smith, K.; Lindsay, C. M.; Pritchard, G. J. J. Am. Chem. Soc. 1989, 111, 665-669.

(6) Zou, L. H.; Reball, J.; Mottweiler, J.; Bolm, C. Chem. Commun. 2012, 48, 11307-11309.

(7) O’Mahony, G. E.; Ford, A.; Maguire, A. R. J. Org. Chem. 2012, 77, 3288-3296.

(8) Horner, L.; Lawson, A. J.; Simons, G. Phosphorus and Sulfur, 1982, 12, 353-356.

(9) Cabiddu, M. G.; Cabiddu, S.; Cadoni, E.; Montis, S. D.; Fattuoni, C.; Melis, S. Tetrahedron, 2004, 60, 3915-3920.

(10) Shanks, D.; Amorati, R.; Fumo, M. G.; Pedulli, G. F.; Valgimigli, L.; Engman, L. J. Org. Chem. 2006, 71, 1033-1038. 
Table S1. Inhibited Rates of Conjugated Diene Formation $\left(\boldsymbol{R}_{\mathrm{inh}}\right)$ and Inhibition Times $\left(T_{\text {inh }}\right)$ with Acidified DTT (0.5 mM)

\begin{tabular}{|c|c|c|}
\hline \multirow{2}{*}{$\begin{array}{l}\text { Antioxidant } \\
\qquad(40 \mu \mathrm{M})\end{array}$} & \multicolumn{2}{|c|}{ with $\mathrm{DTT}^{a}$} \\
\hline & $R_{\mathrm{inh}}^{b}(\mu \mathrm{M} / \mathrm{h})$ & $T_{\mathrm{inh}}^{c}(\min )$ \\
\hline $\mathbf{6 a}$ & $43 \pm 1$ & $67 \pm 4$ \\
\hline $6 b$ & $32 \pm 3$ & $83 \pm 6$ \\
\hline $6 c$ & $27 \pm 3$ & $72 \pm 6$ \\
\hline $6 d$ & 293 & 0 \\
\hline $7 \mathbf{a}$ & $19 \pm 1$ & $100 \pm 5$ \\
\hline $7 b$ & $18 \pm 2$ & $109 \pm 7$ \\
\hline $7 c$ & $17 \pm 1$ & $121 \pm 3$ \\
\hline $7 d$ & 303 & 0 \\
\hline $9 \mathbf{a}$ & $18 \pm 2$ & $99 \pm 4$ \\
\hline $9 b$ & 417 & 0 \\
\hline $\mathbf{1 1}^{d}$ & $58 \pm 4$ & $64 \pm 6$ \\
\hline $13^{d}$ & $36 \pm 3$ & $83 \pm 4$ \\
\hline $\mathrm{Ph}_{2} \mathrm{~S}_{2}$ & 435 & 0 \\
\hline$\alpha-\mathrm{T}$ & $22 \pm 3$ & $103 \pm 8$ \\
\hline
\end{tabular}

${ }^{a} \mathrm{pH}$ was adjusted to 3.3 with $\mathrm{AcOH} .{ }^{b}$ Rate of peroxidation during the inhibited phase. Uninhibited phase rate is 411 $\mu \mathrm{M} / \mathrm{h}$ with acidified DTT. Errors correspond to \pm SD for triplicates. ${ }^{c}$ Inhibited phase of peroxidation. Errors correspond to \pm SD for triplicates. ${ }^{d} 80 \mu \mathrm{M}$ of antioxidant was used in the assay. 
Table S2. Inhibited Rates of Conjugated Diene Formation $\left(\boldsymbol{R}_{\text {inh }}\right)$ and Inhibition Times $\left(T_{\text {inh }}\right)$ with TCEP at Gradient Concentrations

\begin{tabular}{|c|c|c|}
\hline $\begin{array}{l}\text { Concentration of } \\
\text { TCEP }(\mathrm{mM})\end{array}$ & $R_{\mathrm{inh}}^{a}(\mu \mathrm{M} / \mathrm{h})$ & $T_{\mathrm{inh}^{b}}^{b}(\min )$ \\
\hline $0.0625^{c}$ & $67 \pm 4$ & $25 \pm 2$ \\
\hline $0.125^{d}$ & $32 \pm 2$ & $58 \pm 5$ \\
\hline 0.25 & $15 \pm 1$ & $121 \pm 4$ \\
\hline 0.5 & $2.3 \pm 1$ & $324 \pm 5$ \\
\hline 1.0 & $2.5 \pm 0$ & $572 \pm 6$ \\
\hline \multicolumn{3}{|c|}{$\begin{array}{l}{ }^{a} \text { Rate of peroxidation during the inhibited phase. Errors } \\
\text { correspond to } \pm \text { SD for triplicates. }{ }^{b} \text { Inhibited phase of } \\
\text { peroxidation. Errors correspond to } \pm \text { SD for triplicates. }{ }^{c} \mathrm{pH} \\
(=4.3) \text { was adjusted to } 3.3 \text { with } \mathrm{AcOH} .{ }^{d} \mathrm{pH}(=4.1) \text { was } \\
\text { adjusted to } 3.3 \text { with } \mathrm{AcOH} \text {. }\end{array}$} \\
\hline
\end{tabular}


${ }^{1} \mathrm{H}$ NMR Spectrum of Dioctyl diselenide

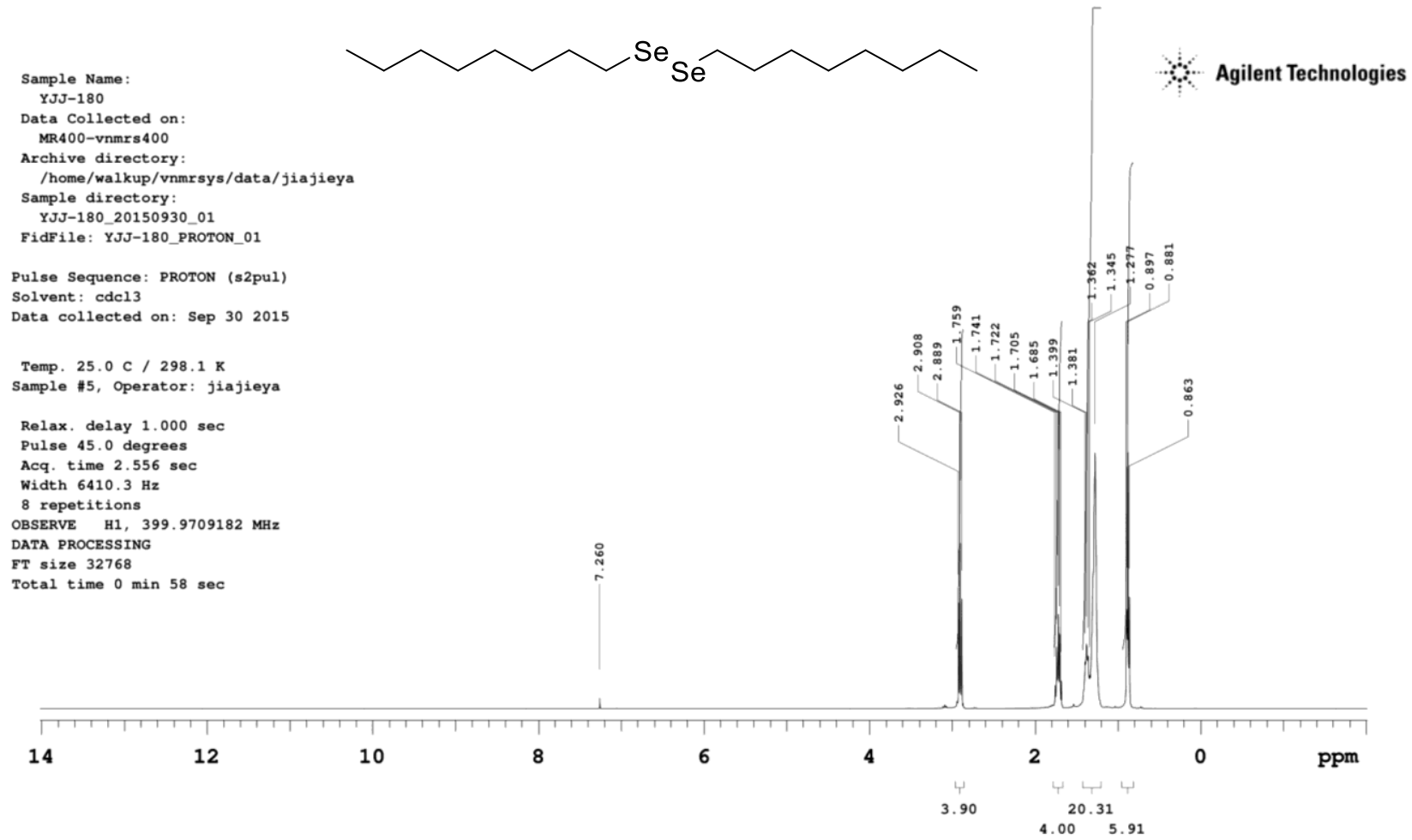

${ }^{13} \mathrm{C}$ NMR Spectrum of Dioctyl diselenide

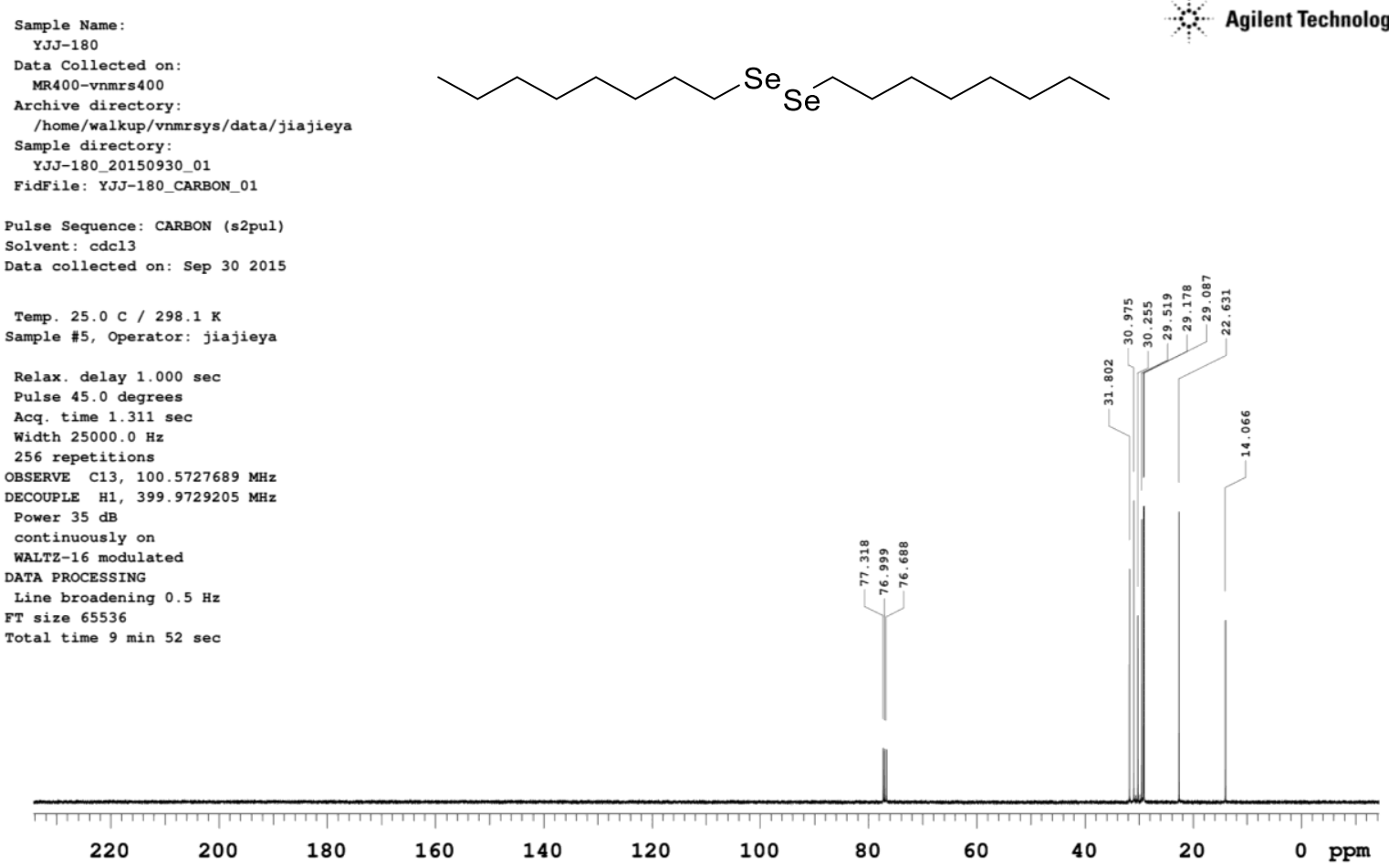


${ }^{77}$ Se NMR Spectrum of Dioctyl diselenide

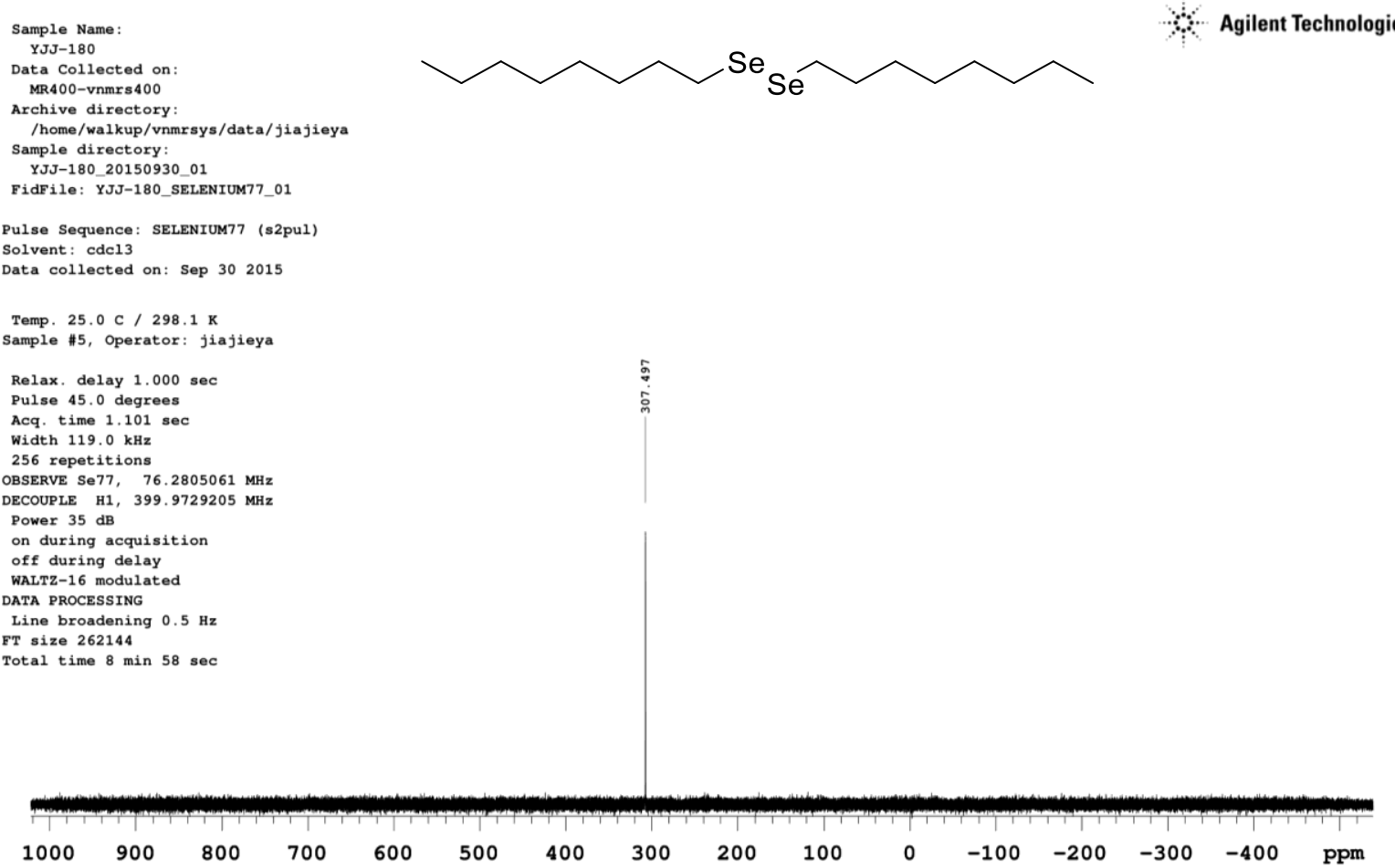

$\begin{array}{llllllllllllllll}1000 & 900 & 800 & 700 & 600 & 500 & 400 & 300 & 200 & 100 & 0 & -100 & -200 & -300 & -400 & \text { ppm }\end{array}$

${ }^{1}$ H NMR Spectrum of Compound $\mathbf{6 a}$

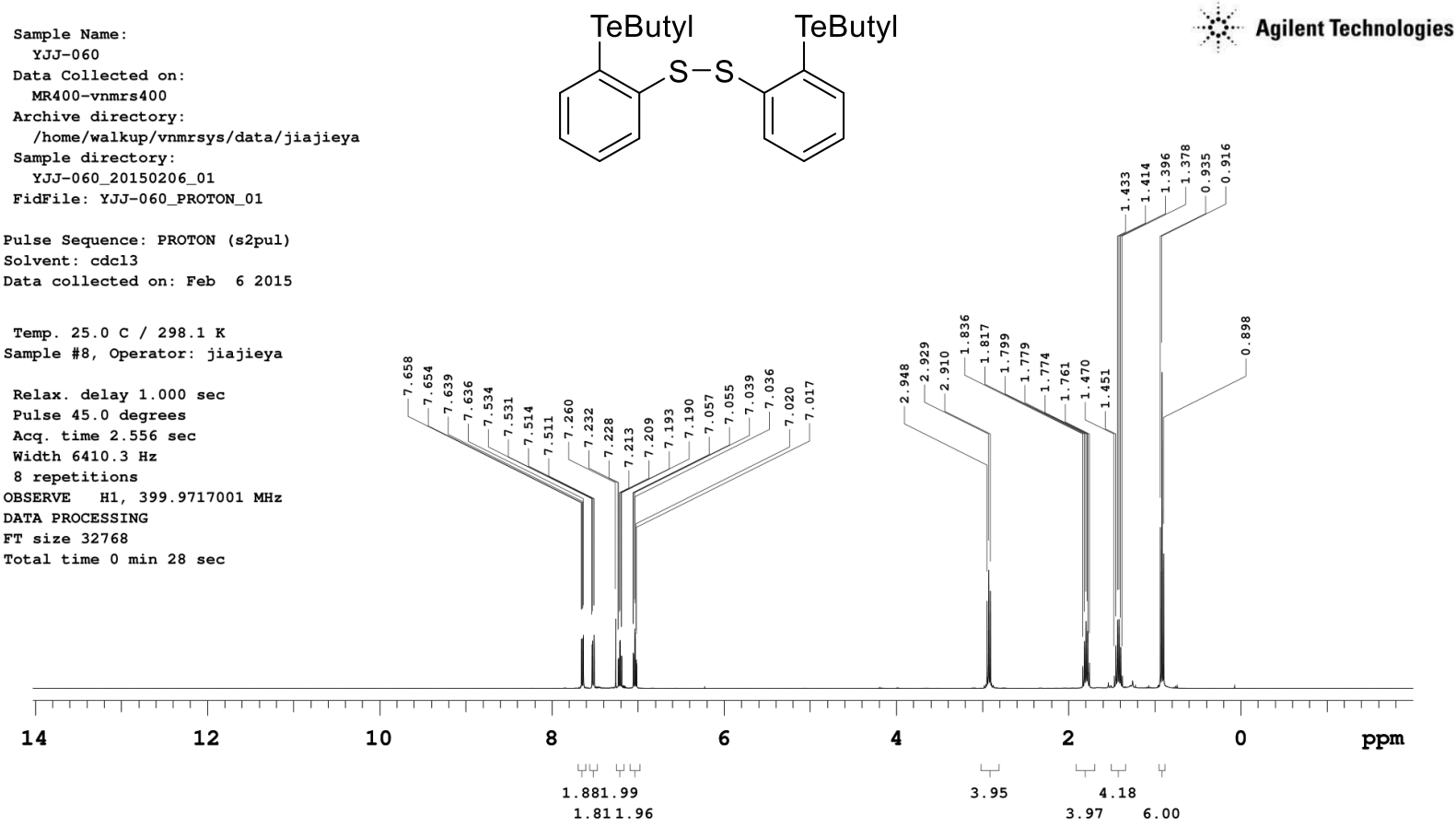


${ }^{13} \mathrm{C}$ NMR Spectrum of Compound $\mathbf{6 a}$

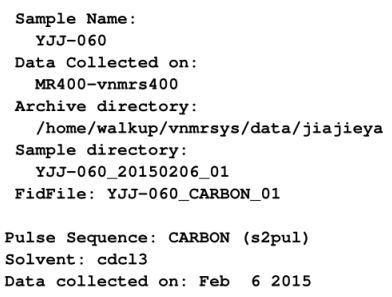

ulse Sequence: CARBON (s2pul) Solvent: cdc13

Data collected on: Feb 62015<smiles>CC(C)(C)CC(C)(C)c1ccccc1SSc1ccccc1C(C)(C)C</smiles>

Temp. $25.0 \mathrm{C} / 298.1 \mathrm{~K}$
Sample \#8, operator: jiajieya

Relax. delay $1.000 \mathrm{sec}$

Pulse 45.0 degrees

Acq. time $1.311 \mathrm{sec}$

Width $25000.0 \mathrm{~Hz}$

256 repetitions

OBSERVE C13，100.5729651 MH

DECOUPLE H1, 399.9737055 MHZ

Power $35 \mathrm{~dB}$

continuously on

WALTZ-16 modulated

DATA PROCESSING

Line broadening $0.5 \mathrm{~Hz}$

FT size 65536

Total time $10 \mathrm{~min}$

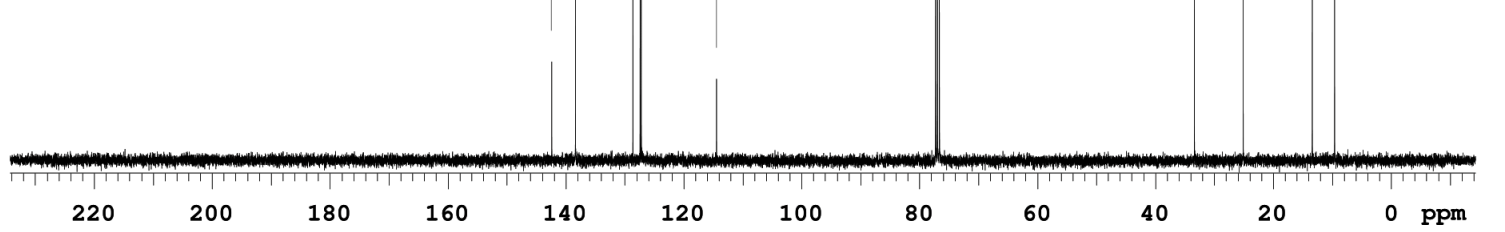

${ }^{125}$ Te NMR Spectrum of Compound $\mathbf{6 a}$

Sample Name:

Data Collected on:

MR400-vnmrs400

Archive directory

/home/walkup/vnmrsys/data/jiajieya

Sample directory:
YJJ-060-Te_20150108_01

FidFile: YJJ-060-Te_TEIIURIUM_01

Pulse Sequence: TELLURIUM (s2pul)

Solvent: cdc13

Dolventilect 82015

Temp. $25.0 \mathrm{C} / 298.1 \mathrm{~K}$

Sample \#6, Operator: jiajieya

Relax. delay $1.000 \mathrm{sec}$

Pulse 61.7 degrees

Acq. time 0.600 se

Width $357.1 \mathrm{kHz}$

8000 repetitions

(26.1849115 MHz

Power $35 \mathrm{~dB}$

WALTZ-16 modulated

Wari 16 modula

Iine broadening

TT size 524288

Total time $3 \mathrm{hr}, 33 \mathrm{~min}$<smiles>CC(C)(C)CC(C)(C)c1ccccc1SSc1ccccc1C(C)(C)C</smiles>

Agilent Technologies

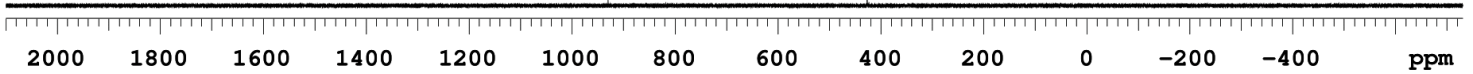


${ }^{1} \mathrm{H}$ NMR Spectrum of Compound $\mathbf{6} \mathbf{b}$

Sample Name

Data Collected on:

MR400-vnmrs400

/home/walkup/vnmrsys/data/jiajieya

Sample di rectory:

FidFile: YJJ-110-H_PROTON_01

Pulse Sequence: PROTON (s2pul)

Solvent: cdc13

Data collected on: Jan 292015

Temp. $25,0 \mathrm{C} / 298,1 \mathrm{x}$

Sample \#5, Operator: jiajieya

Relax. delay $1.000 \mathrm{sec}$

Pulse 45.0 degrees

Acq. time $2.556 \mathrm{sec}$

Width $6410.3 \mathrm{~Hz}$

3 repetitions

OBSERVE H1, 399.9717000 MHZ

DATA PROCESSING

FT size 3276

Total time 0 min $58 \mathrm{sec}$<smiles></smiles>

Agilent Technologies

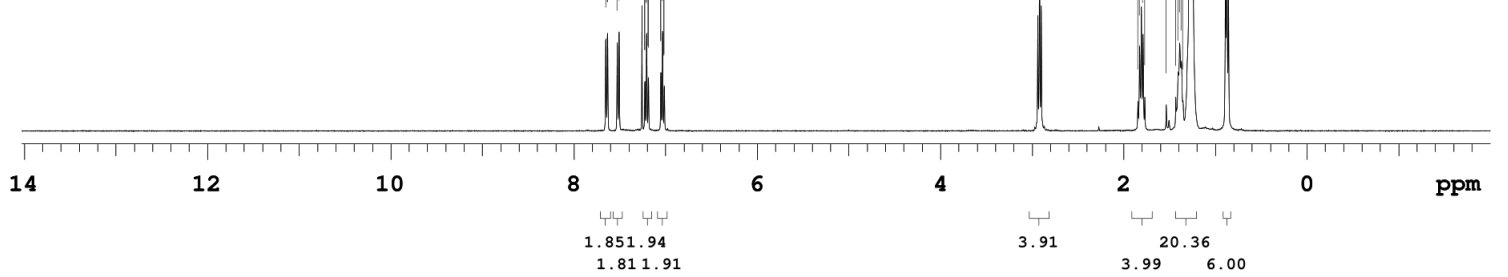

${ }^{13} \mathrm{C}$ NMR Spectrum of Compound $\mathbf{6 b}$

Sample Name:

YJJ-110

Data Collected on:

MR400-vnmrs400

chive directory:

/home/walkup/vnmrsys/data/jiajieya

Sample directory:

FidFile: YJJ-110 CARBON 01

Pulse Sequence: CARBON (s2pul)

Solvent: cdc13

Data collected on: Jan 292015

Temp. $25.0 \mathrm{C} / 298.1$

sample \#2, Operator: jiajieya

Relax. delay $1.000 \mathrm{sec}$

Pulse 45.0 degrees

Width $25000.0 \mathrm{~Hz}$

सc $25000.0 \mathrm{~Hz}$

OBSERVE C13，100.5729651 MHz

DECOUPLE H1, 399.9737055 MHz

Power $35 \mathrm{di}$

Power 35 dB

WALTZ-16 modulated

DATA PROCESSING

Line broadening $0.5 \mathrm{~Hz}$

FT size 65536

Total time $12 \mathrm{~min}$<smiles></smiles>

Agilent Technologies

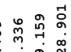

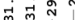

1 \%

โ

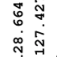
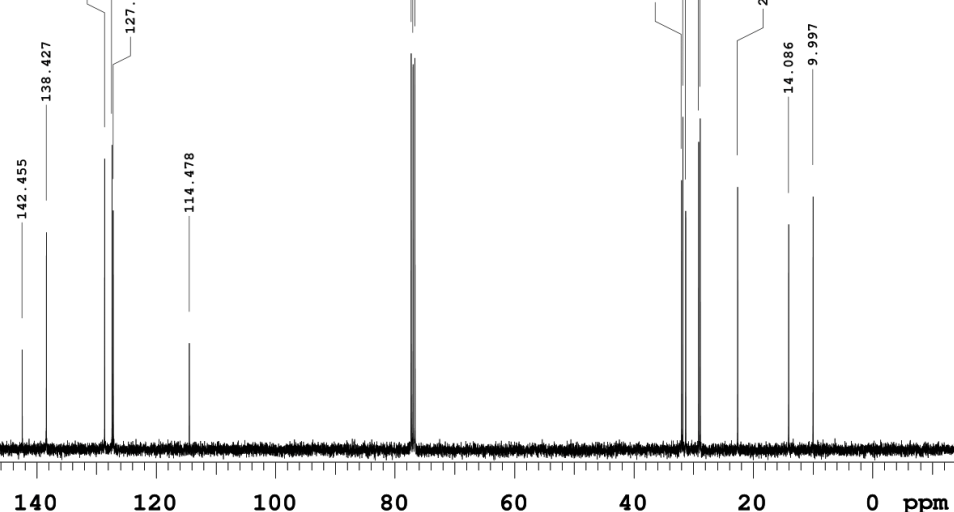


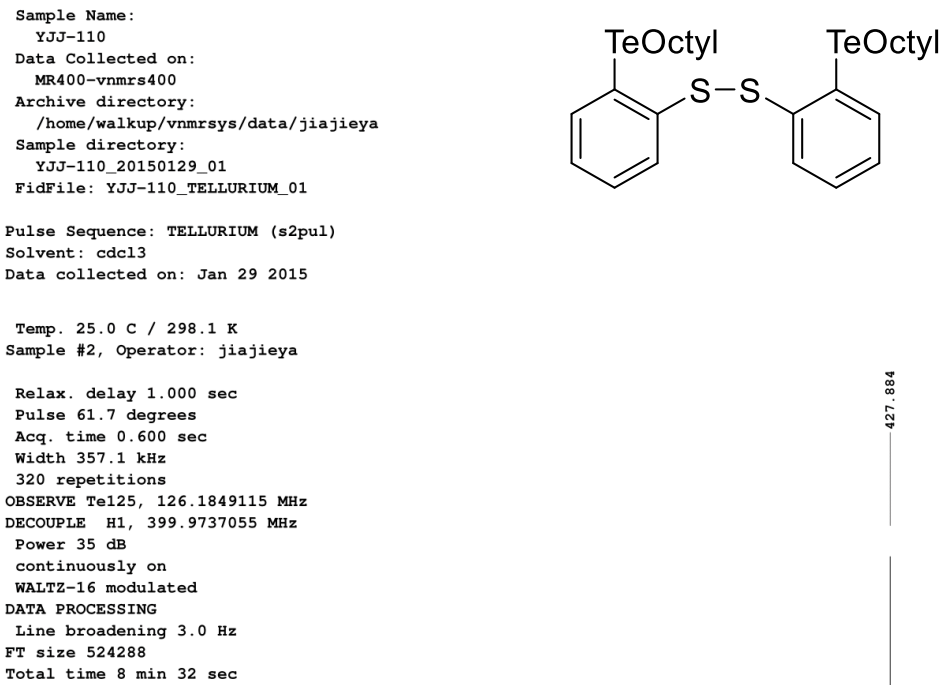

Pulse Sequence: TELLURIUM (s2pul)

Solvent: cdc13

Data collected on: Jan 292015

Temp. $25.0 \mathrm{C} / 298.1 \mathrm{~K}$

Sample \#2, Operator: jiajieya

Relax. delay $1.000 \mathrm{sec}$

Pulse 61.7 degrees

Acq. time 0.600 sec

width $357.1 \mathrm{kHz}$

320 repetitions

OBSERVE Te125, $126.1849115 \mathrm{MH}$

DECOUPLE H1，399.9737055 MHz

Power $35 \mathrm{~dB}$

continuously on

WALTZ-16 modulated

DATA PROCESSING

Line broadening $3.0 \mathrm{~Hz}$

$32 \mathrm{sec}$

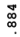

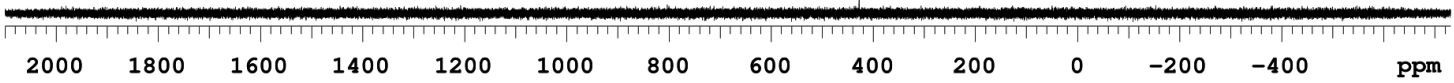

${ }^{1}$ H NMR Spectrum of Compound $\mathbf{6 c}$

Sample Name:

Data Collected on

MR400-vnmrs400

/home/walkup/vnmrsys/data/jiajieya

Sample directory:

YJJ-082-H_20150202_02

FidFile: YJJ-082-H_PROTON_01

Pulse Sequence: PROTON (s2pul)

Solvent: cdc13

Data collected on: Feb 22015

Temp. $25.0 \mathrm{C} / 298.1 \mathrm{~K}$

Sample \#4, Operator: jiajieya

Relax. delay $1.000 \mathrm{sec}$

Pulse 45.0 degrees

Acq. time $2.556 \mathrm{sec}$

Width $6410.3 \mathrm{~Hz}$

repetitions

DATA PROCESSING

FT size 65536

Total time 0 min $58 \mathrm{sec}$ 
${ }^{13} \mathrm{C}$ NMR Spectrum of Compound $\mathbf{6 c}$

Sample Name

YJJ-082

Data Collected on

MR400-vnmrs400

Archive directory

/home/walkup/vnmrsys/data/jiajieya

Sample directory:

FidFile: YJJ-082_CARBON_01

Pulse Sequence: CARBON (s2pul)

Solvent: cdc13

Data collected on: Feb 32015

Temp. $25.0 \mathrm{C} / 298.1 \mathrm{~K}$

Sample \#8, Operator: jiajieya

Relax. delay $1.000 \mathrm{sec}$

Pulse 45.0 degrees

Acq. time $1.311 \mathrm{sec}$

Width $25000.0 \mathrm{~Hz}$

OBSERVE C13, 100.5729640 MHz

OBSERVE C13, $100.5729640 \mathrm{MHz}$

Power $35 \mathrm{~dB}$

nuously on

WALTZ-16 modulated

Line broadening $0.5 \mathrm{~Hz}$

Total time $1 \mathrm{hx}, 17 \mathrm{~min}$
TeHexadecyl TeHexadecyl<smiles>Cc1ccccc1SSc1ccccc1I</smiles>

$\stackrel{\infty}{m}$
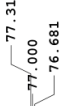

ì

Agilent Technologies
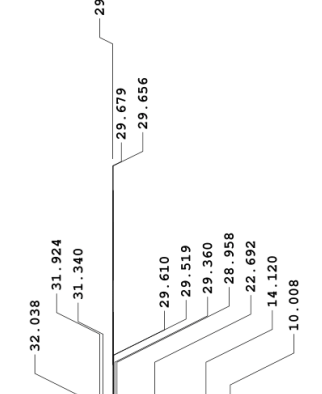

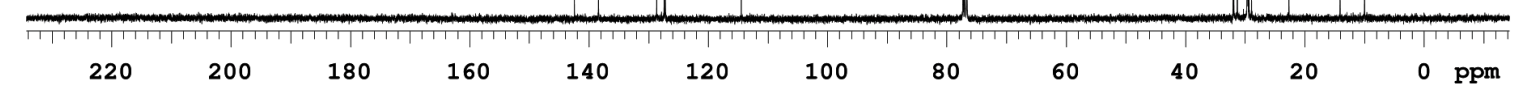

${ }^{125}$ Te NMR Spectrum of Compound $\mathbf{6 c}$

Sample Name:

YJJ-082-Te

Data Collected on:

MR400-vnmrs400

Archive directory:

/home/walkup/vnmrsys/data/jiajieya

Sample directory:
YJJ-082-Te_20150108 01

YJJ-082-Te_20150108_01
FidFile: YJJ-082-Te_TELLURIUM_01

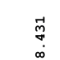

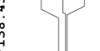

๙ู

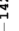

ppm

Pulse Sequence: TELLURIUM (s2pul)

Data collected on: Jan 82015

Temp. $25.0 \mathrm{C} / 298.1 \mathrm{~K}$

Sample \#7, Operator: jiajieya

Relax. delay $1.000 \mathrm{sec}$

Pulse 61.7 degrees

Acq. time 0.600

Width $357.1 \mathrm{kHz}$

OBSERVE Te125, 126.1849115 MHZ

DECOUPLE $125,399.9737055$ MHZ

Dower $35 \mathrm{~dB}$

continuously on

WALTZ-16 modulated

DATA PROCESSING

Line broadening $3.0 \mathrm{~Hz}$

FT size 524288
Total time $3 \mathrm{hr}, 33 \mathrm{~min}$

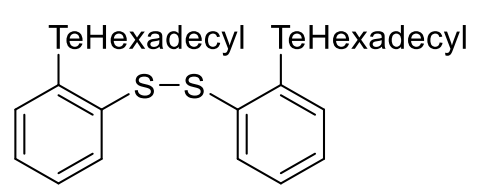

Agilent Technologies

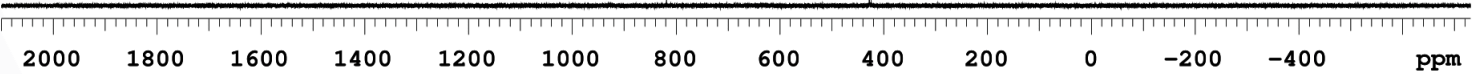


${ }^{1}$ H NMR Spectrum of Compound $\mathbf{6 d}$
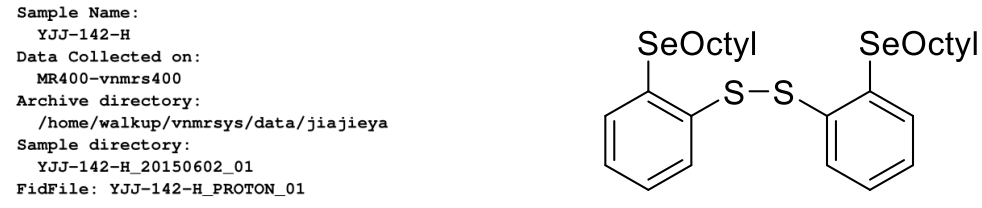

Agilent Technologies

Pulse Sequence: PROTON (s2pul)

Solvent: cdc13

Data collected on: Jun 22015

Temp. $25.0 \mathrm{C} / 298.1 \mathrm{~K}$

Sample \#11, Operator: jiajieya

Relax. delay $1.000 \mathrm{sec}$

Pulse 45.0 degrees

Acq. time $2.556 \mathrm{sec}$

Width $6410.3 \mathrm{~Hz}$

8 repetitions

DATA PROCESSING

FT size 32768

Total time $0 \mathrm{~min} 58 \mathrm{sec}$

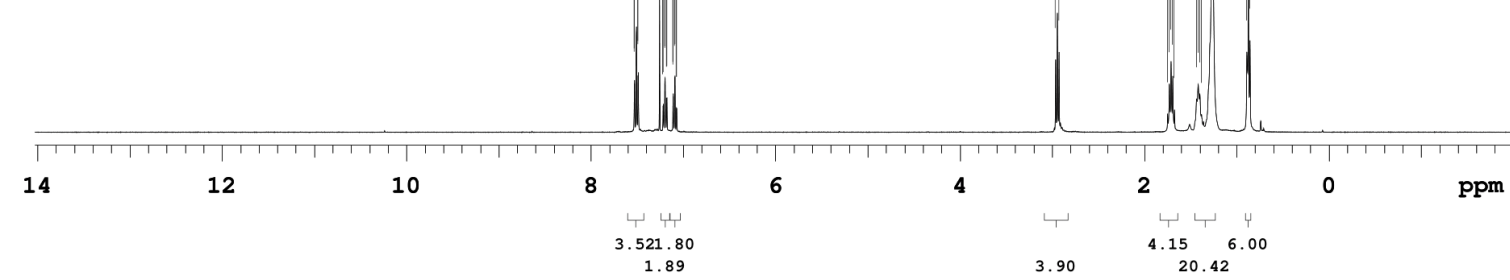

${ }^{13} \mathrm{C}$ NMR Spectrum of Compound $\mathbf{6 d}$

Sample Name:
YJJ-142-C

YJJ-142-C
Data Collected on:

MR400-vnmrs 400

Archive directory:

/home/walkup/vnmrsys/data/jiajieya

Sample directory:

FidFile: YJJ-142-C_CARBON_01

Pulse Sequence: CARBON (s2pul)

Solvent: cdc13

Dolvent: cacis Cant apr 282015

Temp. $25.0 \mathrm{C} / 298.1 \mathrm{~K}$

Sample \#7, Operator: jiajieya

Relax. delay $1.000 \mathrm{sec}$

Pulse 45.0 degrees

Acq. time 1.311 se

Width $25000.0 \mathrm{~Hz}$

OBSERVE C13, 100.5729070 MH

OBSERVE C13, $100.5729070 \mathrm{MHZ}$

Power $35 \mathrm{~dB}$

WALT2-16 modulated

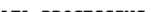

Iine broadening

Total time $20 \mathrm{~min}$<smiles>COS(=O)(=O)c1ccccc1SSc1ccccc1S(=O)(=O)O[Na]</smiles>

Agilent Technologies

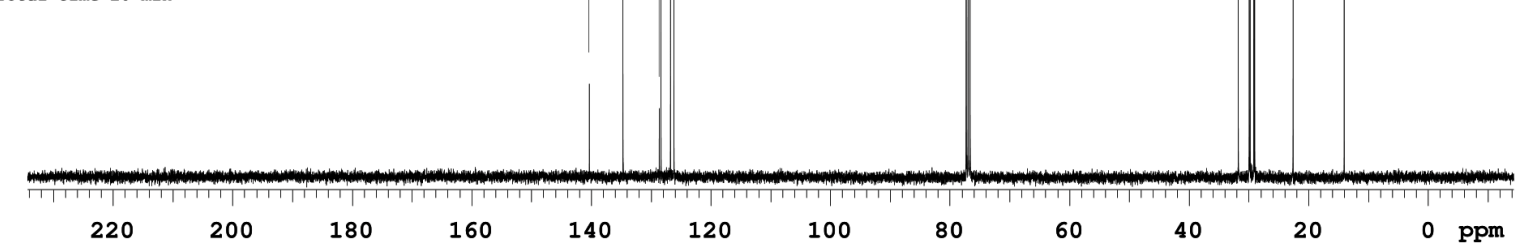


Sample Name:

Agilent Technologies

YJJ-142-Se

Data Collected on

MR400-vnmrs400
Archive directory:

Archive directory:

Sample directory:

YJJ-142-Se_20150428_01

FidFile: YJJ-142-Se_SELENIUM77_01

Pulse Sequence: SELENIUM77 (s2pul)

Solvent: cdcl3
Data collected on: Apr 282015<smiles>O=S(=O)(O)c1ccccc1SSc1ccccc1S(=O)(=O)O[Na]</smiles>

Temp. $25.0 \mathrm{C} / 298.1 \mathrm{~K}$

Sample \#10, Operator: jiajieya

Relax. delay $1.000 \mathrm{sec}$

Pulse 45.0 degrees

Acq. time 1.101 se

256 repetitions

OBSERVE Se77, 76.2806126 MHz

DECOUPIE H1, 399. 9734788 MHZ

Power $35 \mathrm{~dB}$

on during acquisition

off during delay

WALTZ-16 modulat

DATA PROCESSING

Line broadening $0.5 \mathrm{~Hz}$

FT size 262144

Total time 9 min $27 \mathrm{sec}$
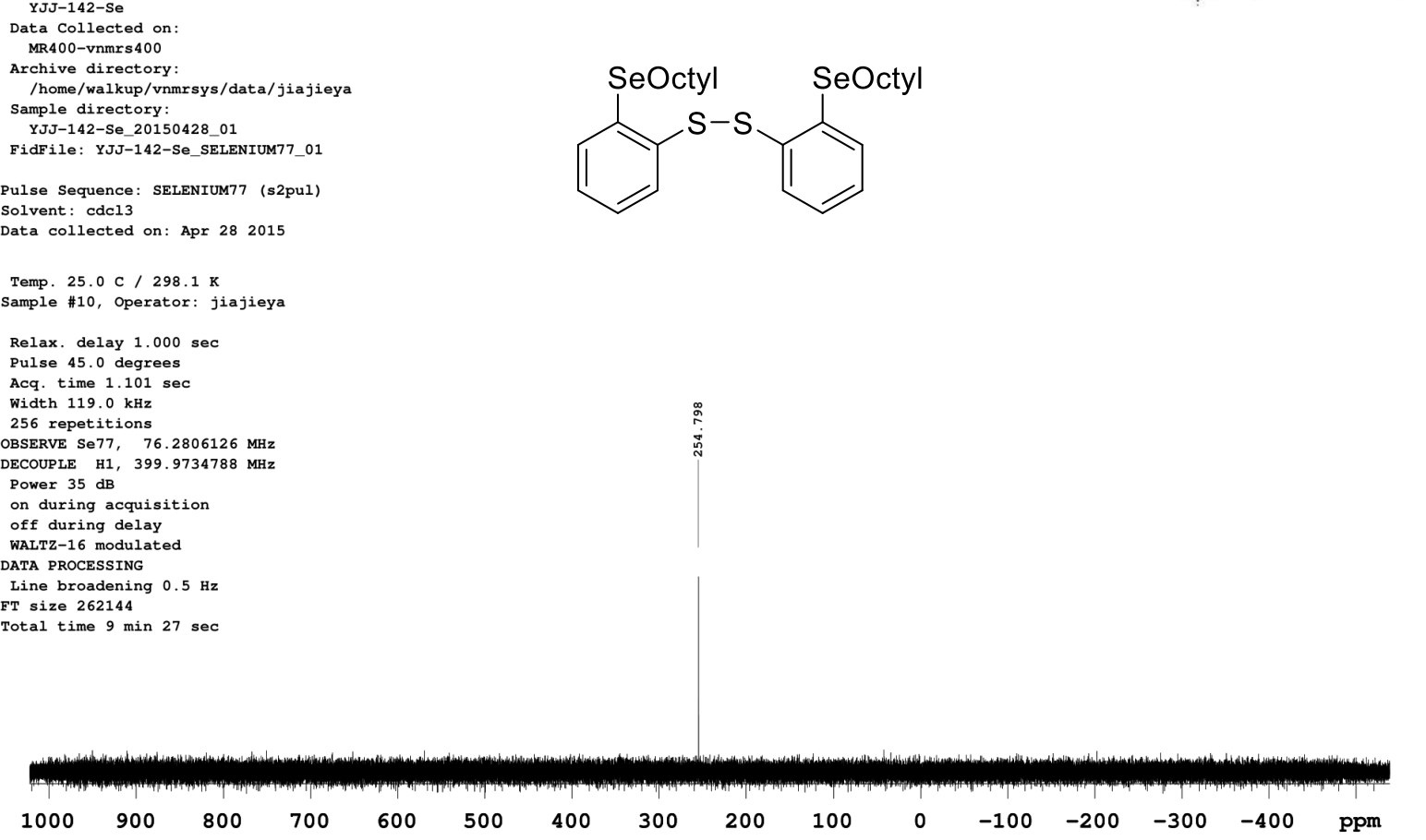

${ }^{1} \mathrm{H}$ NMR Spectrum of Compound 7a

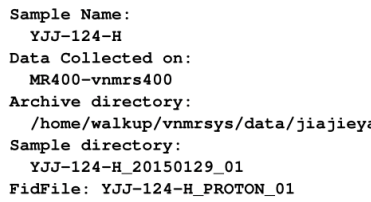

Pulse Sequence: PROTON (s2pul)

Temp. $25.0 \mathrm{C} / 298.1 \mathrm{~K}$

Sample \#6, operator: jiajieya

Relax. delay $1.000 \mathrm{sec}$

Pulse 45.0 degrees

Acq. time 2.556 sec

8 repetitions

OBSERVE H1, $399.9716999 \mathrm{MHz}$

DATA PROCESSING

FT size 65536

Total time $0 \mathrm{~min} 58 \mathrm{sec}$<smiles>CCCCCc1ccc(SSc2ccc([18CH3])cc2)cc1</smiles>

Agilent Technologies
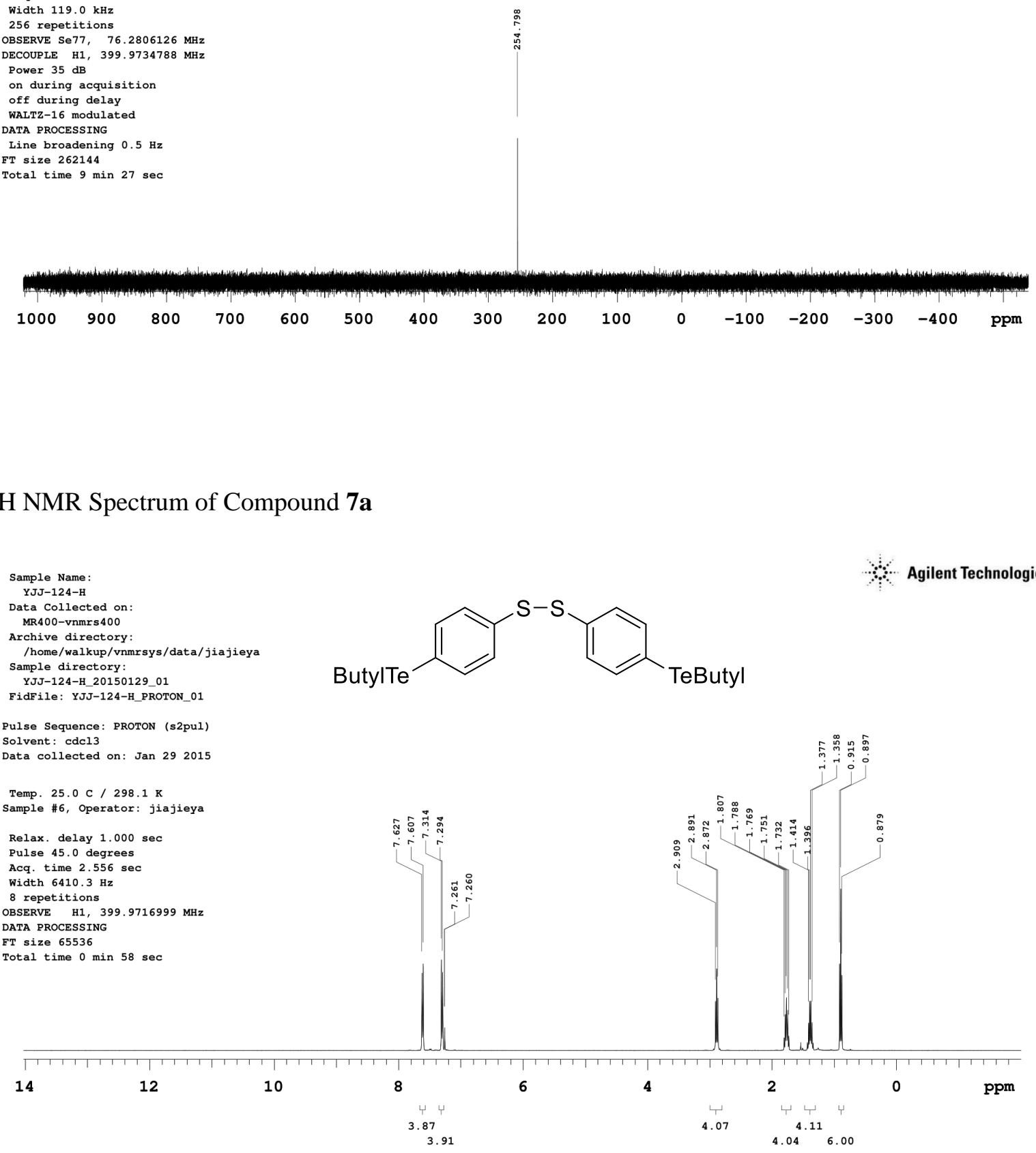
Sample Name:

YJJ-124

Data Collected on:

MR400-vnmrs400
Archive directory:

Archive directory:

Sample directory:

FidFile: YJJ-124_CARBON_01

ButylTe TeButyl

Pulse Sequence: CARBON (s2pul)

Solvent: cdc13

Data collected on: Jan 292015

Temp. 25.0 C / 298.1

Sample \#6, Operator: jiajieya

Relax. delay $1.000 \mathrm{sec}$

Pulse 45.0 degrees

Acq. time 1.311 se

Width $25000.0 \mathrm{~Hz}$

OBSERVE C13, 100.5729655 MHz

DCOU

Power $35 \mathrm{~dB}$

WALTZ-16 modulate

DATA PROCEsSING

Iine broadening $0.5 \mathrm{~Hz}$

FT size 65536

Total time $10 \mathrm{~min}$<smiles>CCCCc1ccc(SSc2ccc(C(CC(C)(C)C)C(C)(C)C)cc2)cc1</smiles>

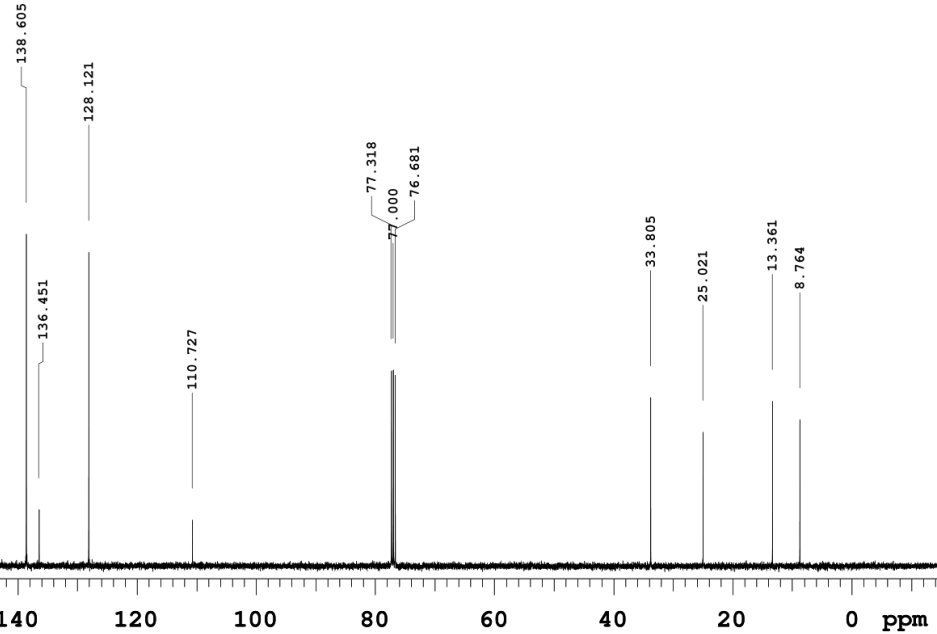

${ }^{125}$ Te NMR Spectrum of Compound 7a

Sample Name:

YJJ-124

Data Collected on

MR400-vnmrs400

Archive directory:

Sample directory

Sample directory:

FidFile: YJJ-124_TELIURIUM_0

Pulse Sequence: TELIURIUM (s2pul)

Solvent: cdc13

Data collected on: Jan 292015

Temp. $25.0 \mathrm{C} / 298.1 \mathrm{x}$

Sample \#6, Operator: jiajieya

Relax. delay $1.000 \mathrm{sec}$

Pulse 61.7 degrees

Acq. time 0.600 sec

Width $357.1 \mathrm{kHz}$

OBSERVE Te125，126.1849115 MHz

B.

Power $35 \mathrm{~dB}$

continuously on

WALTZ-16 modulat

Line broadening 3

Total time 6 min $50 \mathrm{sec}$
Agilent Technologies<smiles>CCCCCc1ccc(SSc2ccc([18CH3])cc2)cc1</smiles>

\begin{tabular}{|c|c|c|c|c|c|c|c|c|c|c|c|}
\hline 2000 & 1800 & 1600 & 1400 & 1200 & 1000 & 80 & 600 & 400 & 200 & 0 & -200 \\
\hline
\end{tabular}


${ }^{1} \mathrm{H}$ NMR Spectrum of Compound $\mathbf{7 b}$

Sample Name:

YJJ-125-H_and_C

Data Collected on
MR400-vnmrs 400

Archive directory:

/home/walkup/vnmrsys/data/jiajieya

Sample directory:

YJJ-125-H_and_C_20150527_0

FidFile: YJJ-125-H_and_C_PROTON_01

Pulse Sequence: PROTON (s2pu1)

Solvent: edc13

Data collected on: May 272015

Temp. $25.0 \mathrm{C} / 298.1 \mathrm{~K}$

Sample \#4, operator: jiajieya

Relax. delay $1.000 \mathrm{sec}$

Pulse 45.0 degrees

Acq. time $2.556 \mathrm{se}$

Width $6410.3 \mathrm{~Hz}$

8 repetitions $399.9714719 \mathrm{MH}$

OBSERVE H1, 39 .

Line broadening $1.0 \mathrm{~Hz}$

Line broaden

Total time 0 min $58 \mathrm{sec}$

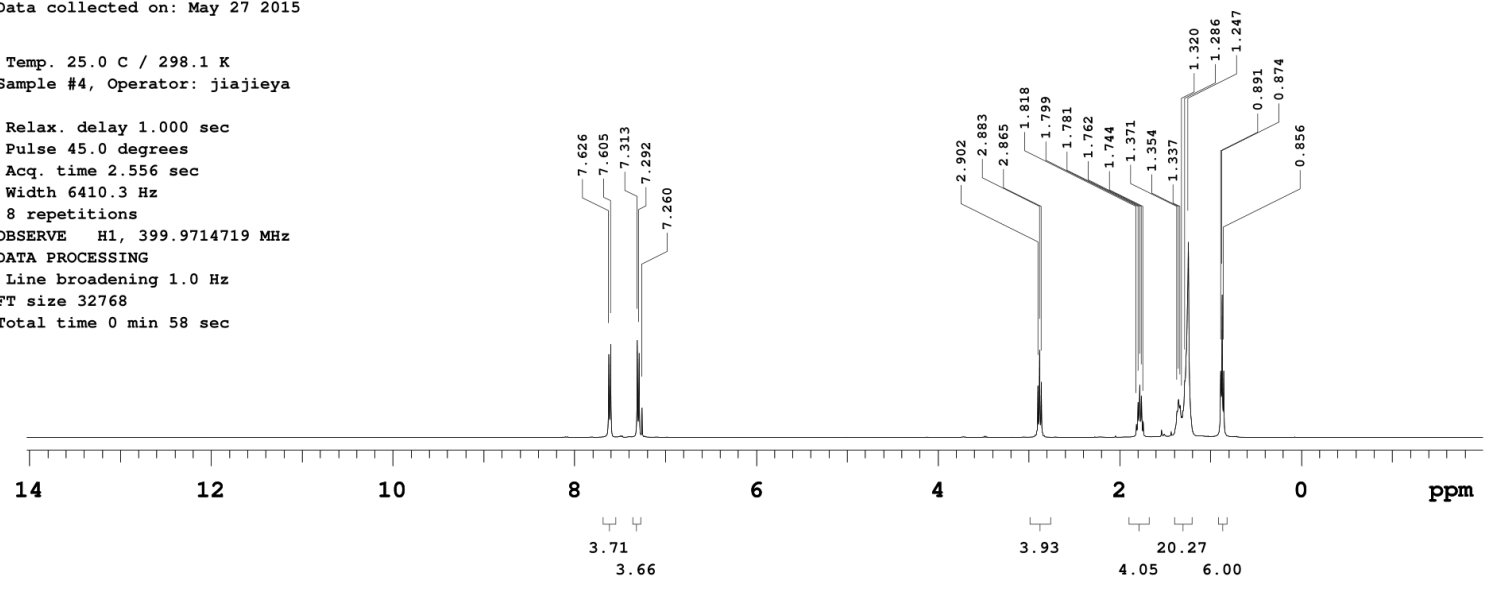

OctylTe

Agilent Technologies

${ }^{13} \mathrm{C}$ NMR Spectrum of Compound $\mathbf{7 b}$

Sample Name:

YJJ-125-H_and_C

Data Collected on

MR400-vnmrs 400

Archive directory:

/home/walkup/vnmisys/data/jiajiega

Sample directory:

YJJ-125-H and 20150527

FidFile: YJJ-125-H_and_C_CARBON_01

Pulse Sequence: CARBON (s2pul)

Solvent: cdcls

Data collected

Temp. $25.0 \mathrm{C} / 298.1 \mathrm{~K}$

Sample \#4, Operator: jiajieya

Relax. delay $1.000 \mathrm{sec}$

Pulse 45.0 degrees

Acq. time $1.311 \mathrm{sec}$

Width $25000.0 \mathrm{~Hz}$

20 repetitions

OBSERVE C13，100.5729078 MHz

DECOUPLE H1, 399.9734788 MHZ

Power $35 \mathrm{~dB}$

continuously on

WALTZ-16 modulated

DATA PROCESSING

Line broadening $0.5 \mathrm{~Hz}$

FT size 65536

Total time $12 \mathrm{~min}$

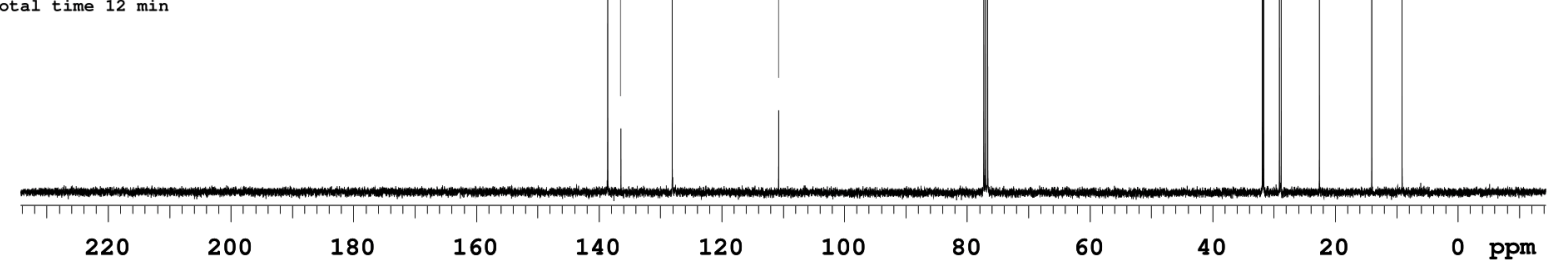


Sample Name:

YJJ-125

Data Collected on:

Archive directory:

/home/walkup/vnmssy/data/jiajieya

Sample directory.

YJJ-125 20150130 01

FidFile: YJJ-125 TELIURIUM 01

Pulse Sequence: TELIURIUM (s2pui)

Solvent: cdc13

Data collected on: Jan 302015

Temp. $25.0 \mathrm{C} / 298.1$

Sample \#2, Operator: jiajieya

Relax. delay $1.000 \mathrm{sec}$

Pulse 61.7 degrees

Acq. time $0.600 \mathrm{sec}$

Width $357.1 \mathrm{kHz}$

OBSERVE Te125, 126.1849115 MHz

DECOUPLE H1, $399.9737055 \mathrm{MHz}$

Power $35 \mathrm{~dB}$

WALTZ-16 modulated

DATA PROCESSING

Line broadening $3.0 \mathrm{~Hz}$

Total time 8 min $32 \mathrm{sec}$
Agilent Technologies

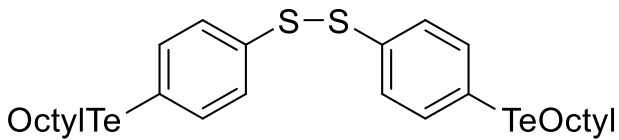

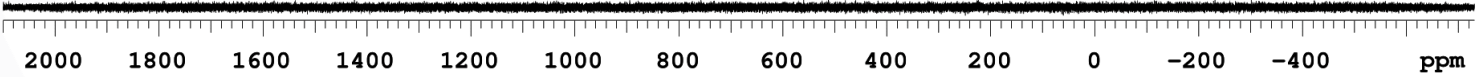

${ }^{1} \mathrm{H}$ NMR Spectrum of Compound 7c

Sample Name:

YJJ-126-H

Data Collected on:

MR400-vnmrs400

/home/nalkup/vn:

Sample directory:

YJJ-126-H_20150602 01

FidFile: YJJ-126-H_PROTON 01

Pulse Sequence: PROTON (s2pul)

Solvent: cdc13

Data collected on: Jun 22015

Temp. $25.0 \mathrm{C} / 298.1 \mathrm{~K}$

Sample \#9, Operator: jiajieya

Relax. delay $1.000 \mathrm{sec}$

Pulse 45.0 degrees

Acq. time $2.556 \mathrm{~s}$

width $6410.3 \mathrm{~Hz}$

8 repetitions

OBSERVE H1, 399.9714718 MH

DATA PROCESSING

FT size 32768

Total time 0 min $58 \mathrm{sec}$
HexadecylTe<smiles>CSSc1ccc(Br)cc1</smiles><smiles>Cc1ccccc1</smiles>

TeHexadecyl

Agilent Technologies

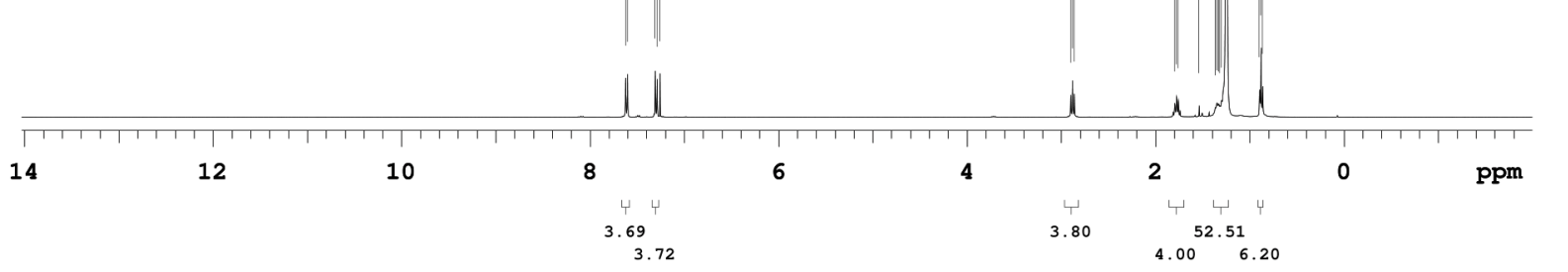


${ }^{13} \mathrm{C}$ NMR Spectrum of Compound 7c

Sample Name:

Data Collected on:

MR400-vnmrs400

Archive directory:

/home/walkup/vnmrsys/data/jiajieya

Sample directory:
YJJ-126-2 20150204

FidFile: YJJ-126-2_CARBON 01

Pulse Sequence: CARBON (s2pul)

Solvent: cdc13

Data collected on: Feb 42015

Temp. $25.0 \mathrm{C} / 298.1$

Sample \#2, Operator: jiajieya

Relax. delay $1.000 \mathrm{sec}$

Pulse 45.0 degrees

Acq. time 1.311 se

Width $25000.0 \mathrm{~Hz}$

OBSERVE C13，100.5729648 MHZ

DECOUPLE H1，399.9737055 MHZ

Power $35 \mathrm{~dB}$

continuously on

WALTZ-16 modulate

DATA PROCESSING

Line broadening $0.5 \mathrm{~Hz}$

FT size 65536

Total time $12 \mathrm{~min}$

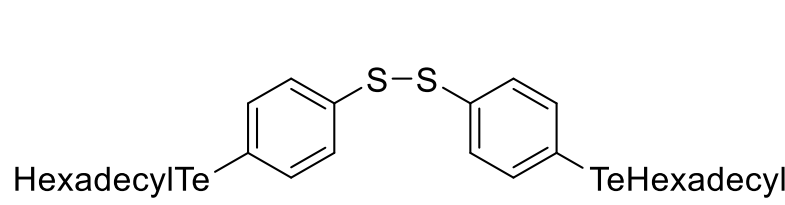

Agilent Technologies

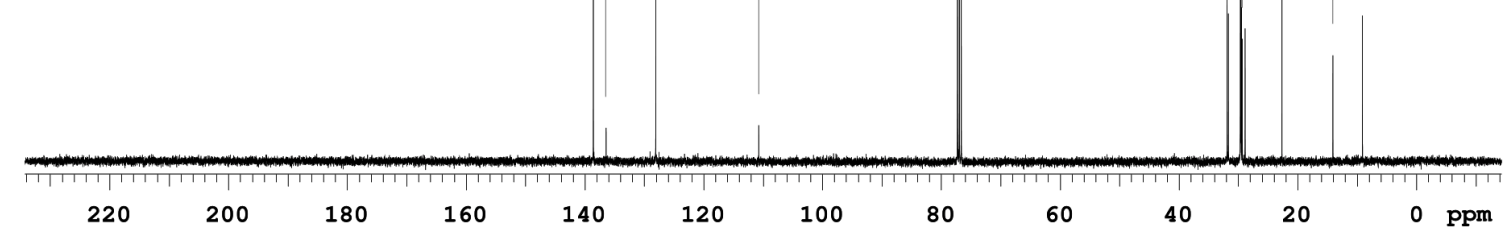

${ }^{125}$ Te NMR Spectrum of Compound $7 \mathbf{c}$

Sample Name:

Data Collected on

MR400-vnmrs400

chive directory

home/walkup/vnmrsys/data/jiajieya

Sample directory:

FidFile: YJJ-126-2_TELLURIUM_01

Pulse Sequence: TELLURIUM (s2pul)

Solvent: cdc13

Data collected on: Feb 42015

Temp. $25.0 \mathrm{C} / 298.1 \mathrm{~K}$

Sample \#2, Operator: jiajieya

Relax. delay $1.000 \mathrm{sec}$

Pulse 61.7 degrees

Acq. time $0.600 \mathrm{sec}$

Width $357.1 \mathrm{kHz}$

320 repetitions
OBSERVE Te125, 126.1849115 MH

OBSERVE Te125, 126.1849115 MHZ

DECOUPLE H1, $399.9737055 \mathrm{MHZ}$

Power $35 \mathrm{~dB}$

WAITz-16 modulated

WALTZ-16 modulated

DATA PROCESSING

$3.0 \mathrm{~Hz}$

Total time 8 min $32 \mathrm{sec}$

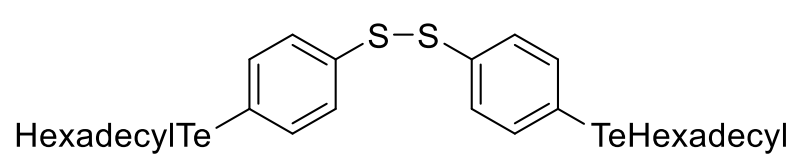

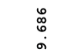

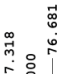

i

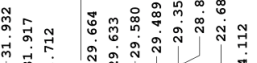
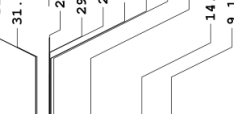

$r$

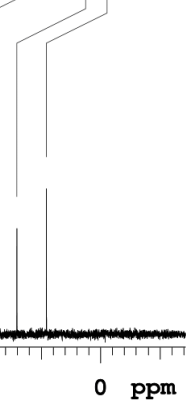


${ }^{1}$ H NMR Spectrum of Compound 7d

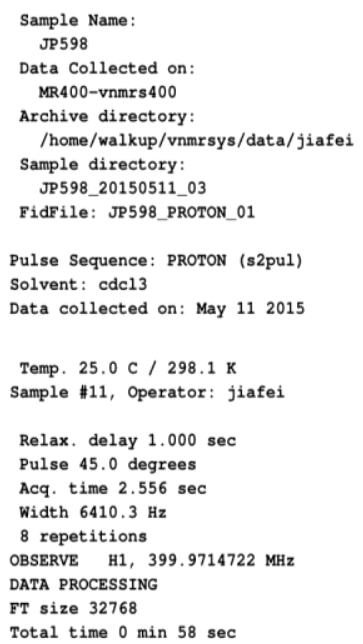

Total time $0 \min 58 \mathrm{sec}$

(n)

ei<smiles>CO[14CH2]O[14CH2]c1ccc(SSc2ccc([Se]O[Na])cc2)cc1</smiles>

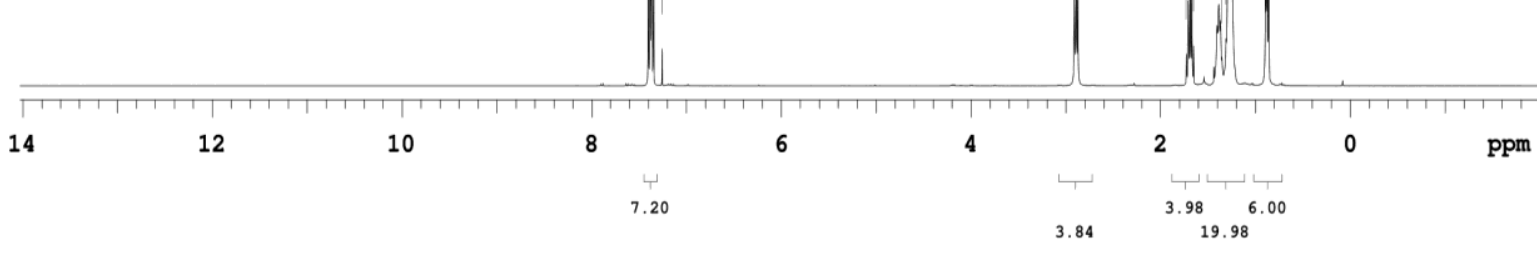

${ }^{13} \mathrm{C}$ NMR Spectrum of Compound 7d

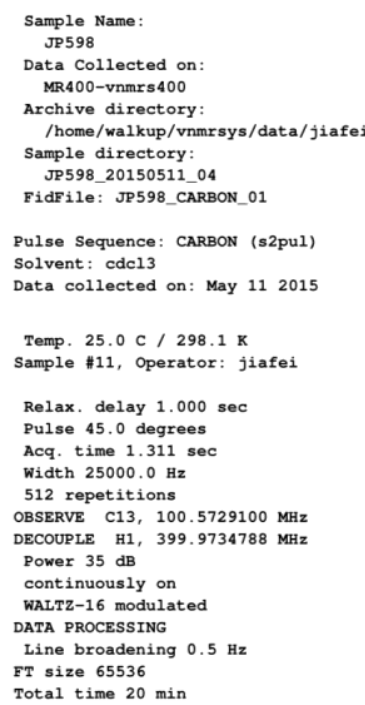


${ }^{77}$ Se NMR Spectrum of Compound 7d

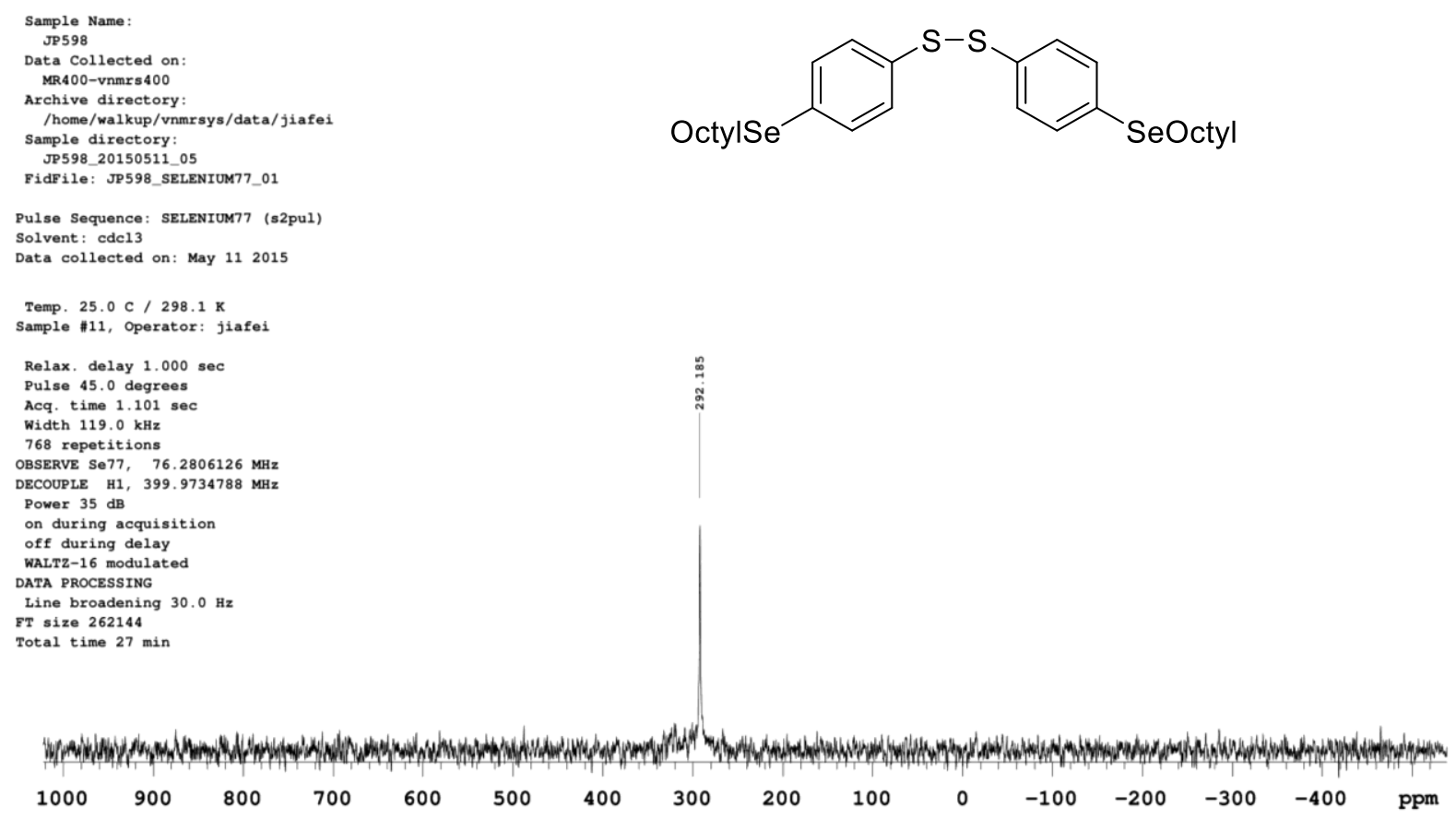

${ }^{1}$ H NMR Spectrum of Compound $\mathbf{8}$

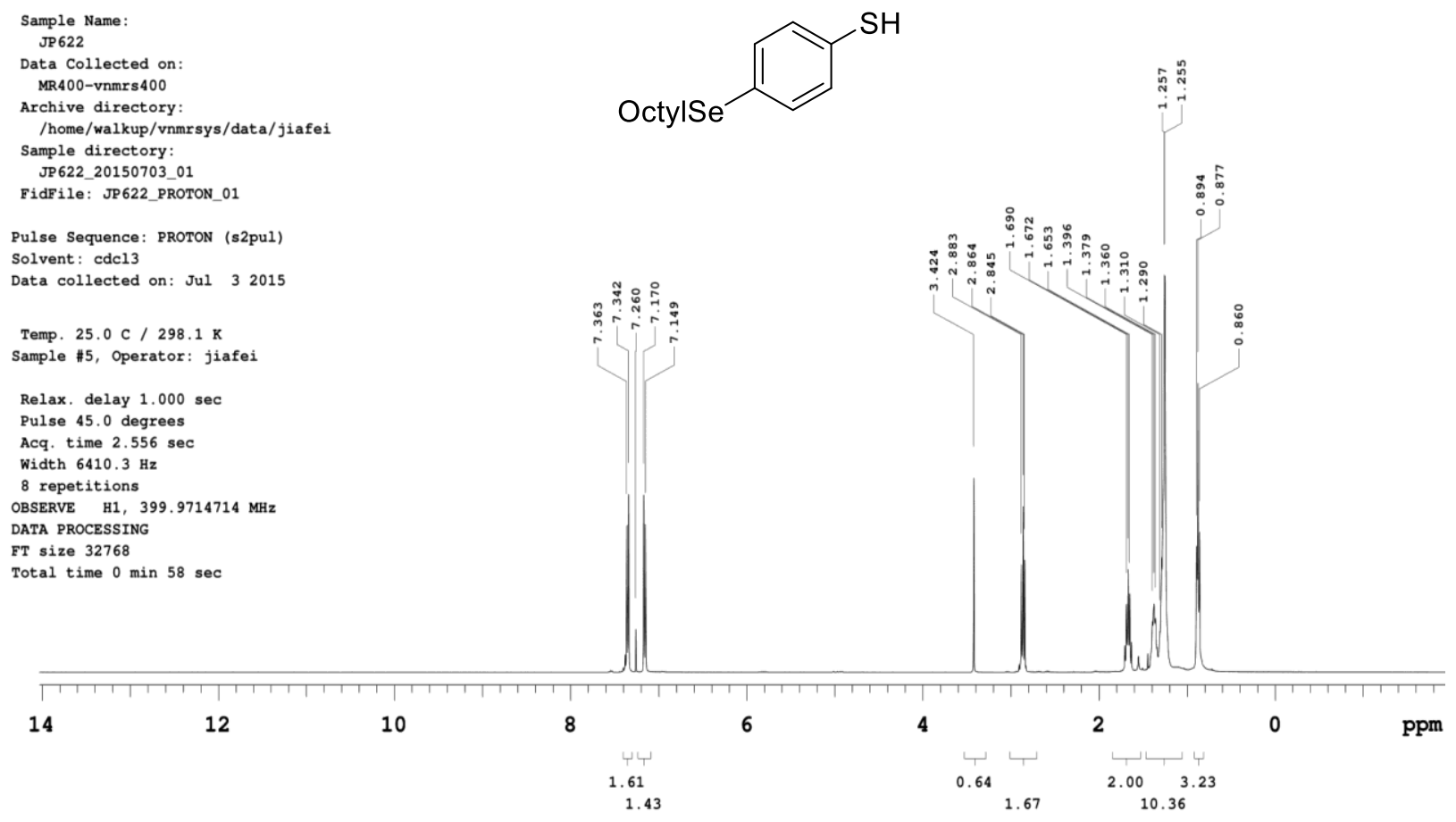


${ }^{13} \mathrm{C}$ NMR Spectrum of Compound $\mathbf{8}$

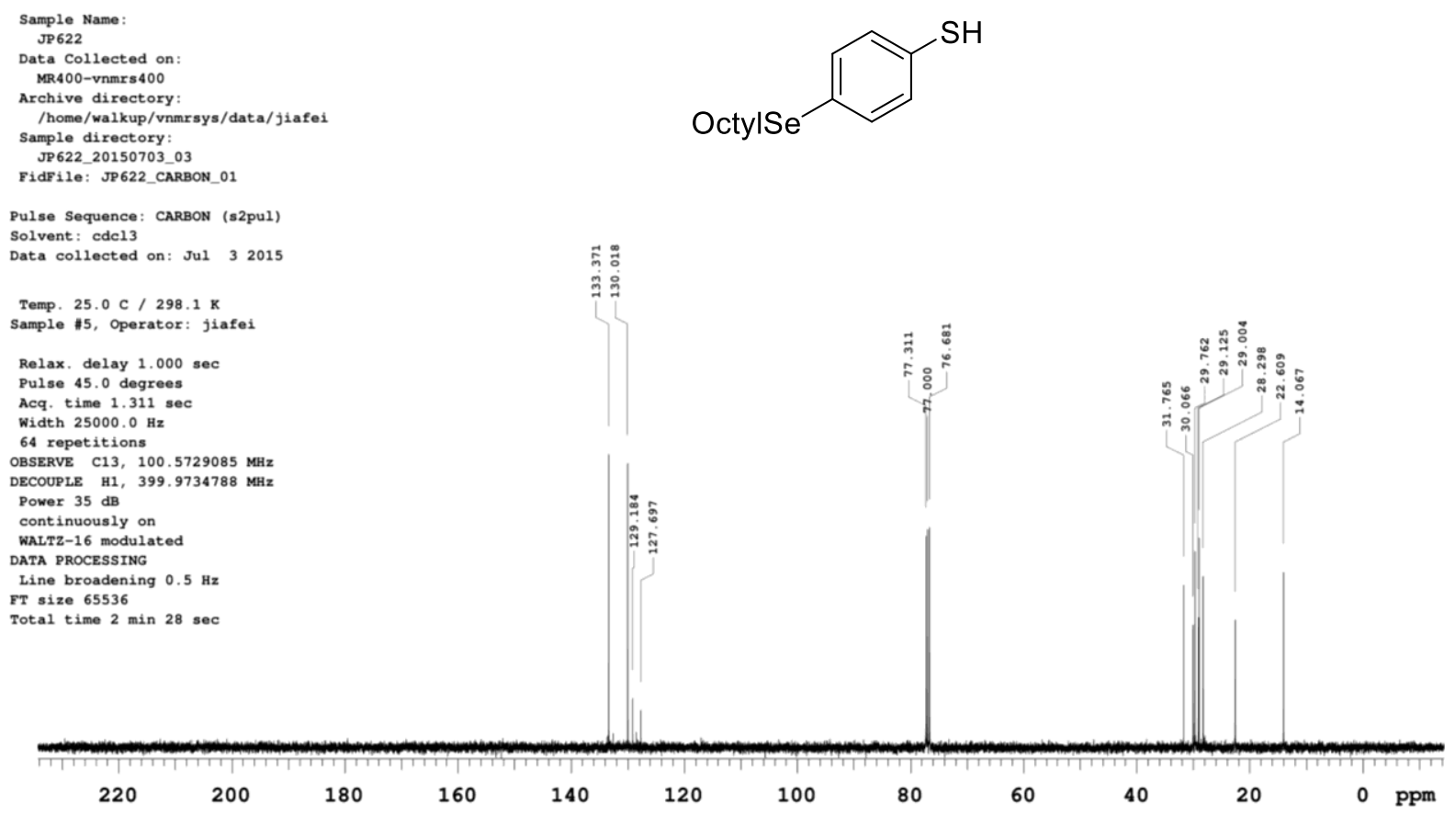

${ }^{77}$ Se NMR Spectrum of Compound $\mathbf{8}$

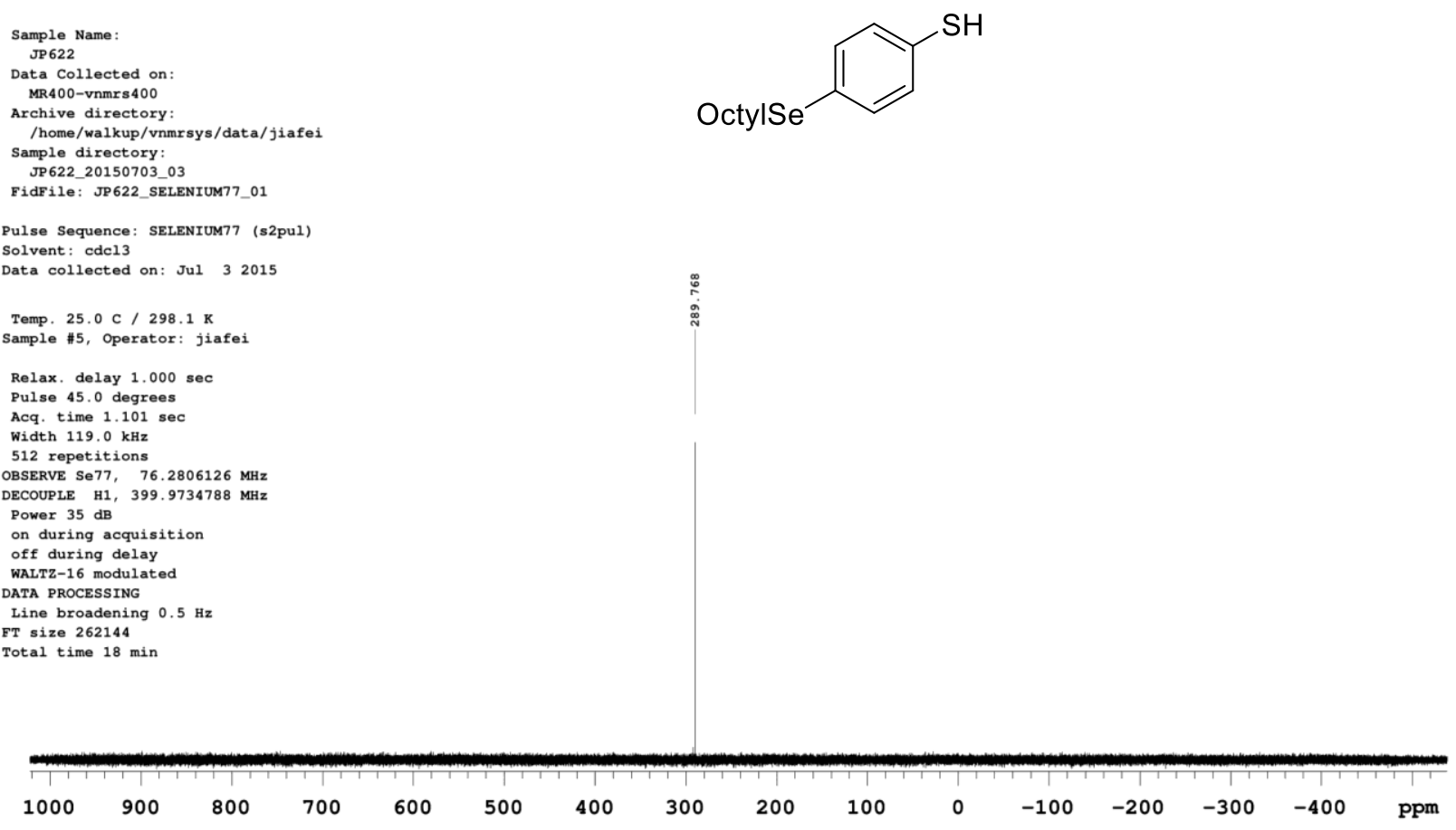


${ }^{1} \mathrm{H}$ NMR Spectrum of Compound 9a

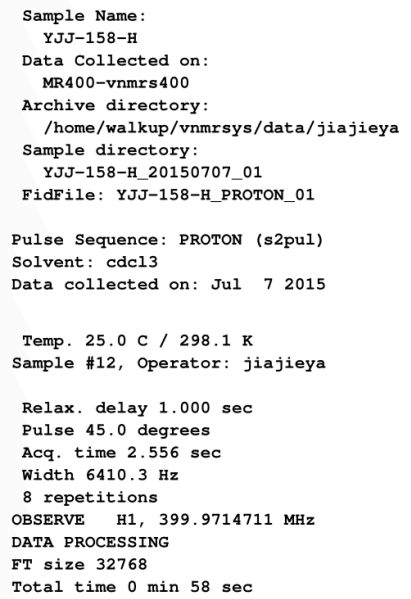

Total time 0 min $58 \mathrm{sec}$<smiles></smiles>

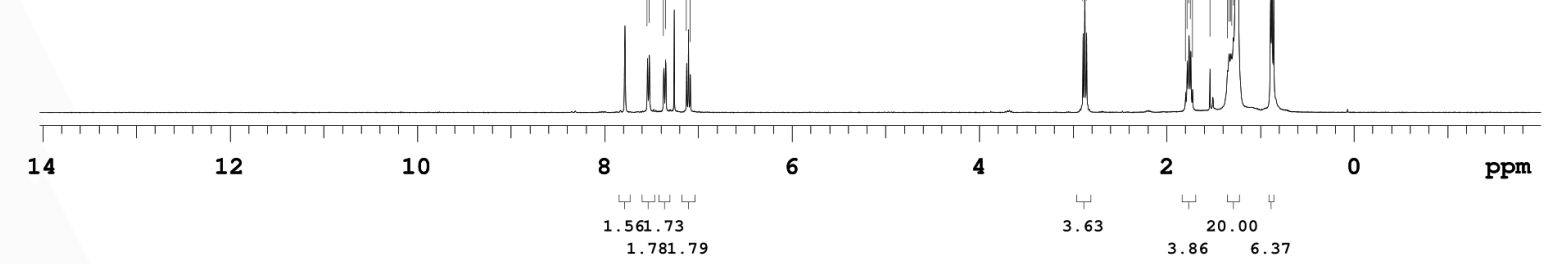

${ }^{13} \mathrm{C}$ NMR Spectrum of Compound $\mathbf{9 a}$

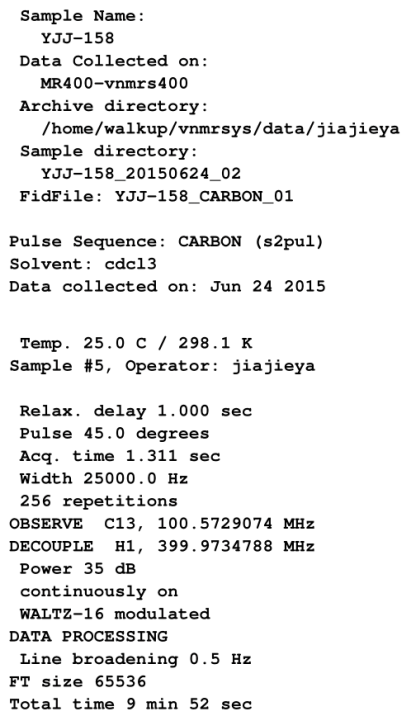

FidFile: YJJ-158_CARBON_01

Pulse Sequence: CARBON (s2pul)

Data collected on: Jun 242015

Temp. $25.0 \mathrm{C} / 298.1 \mathrm{~K}$

Sample \#5, Operator: jiajieya

Relax. delay $1.000 \mathrm{sec}$

Pulse 45.0 degrees

Width $25000 . \mathrm{Hz}$

Width $25000.0 \mathrm{~Hz}$

256 repetitions
OBSERVE C13, 100.5729074 MHz

Power $35 \mathrm{~dB}$

Continuously on

DaTa procissive

DATA DROCESSING 0.5

Line broadening 0.5

Total time 9 min $52 \mathrm{sec}$

eya
$\mathrm{MHz}$
$\mathrm{MHz}$

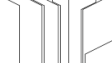

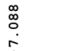<smiles></smiles>

Agilent Technologies

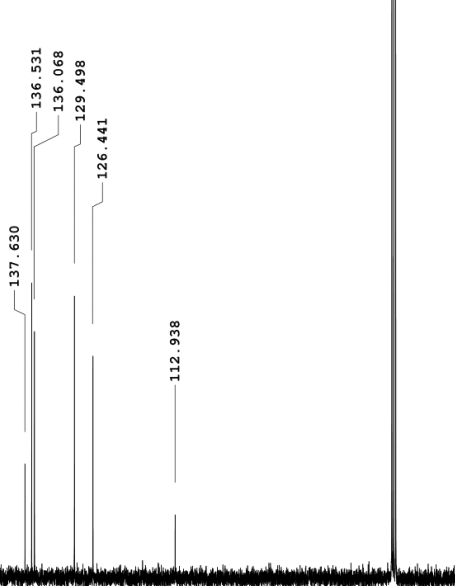

$120 \quad 100$ 
${ }^{125}$ Te NMR Spectrum of Compound $\mathbf{9 a}$

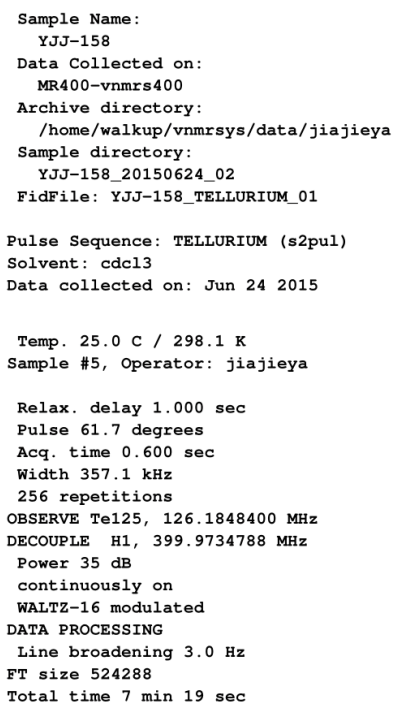<smiles></smiles>

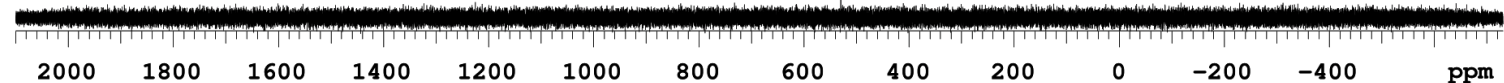

${ }^{1} \mathrm{H}$ NMR Spectrum of 3-(Octylseleno)thiophenol

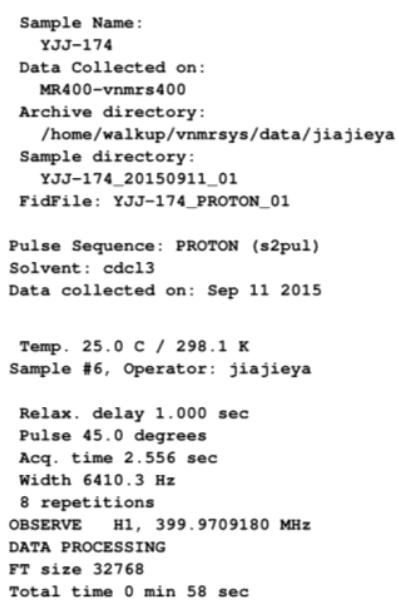

Total time $0 \mathrm{~min} 58 \mathrm{sec}$<smiles>O=S(=O)(O)c1cccc(S)c1</smiles>

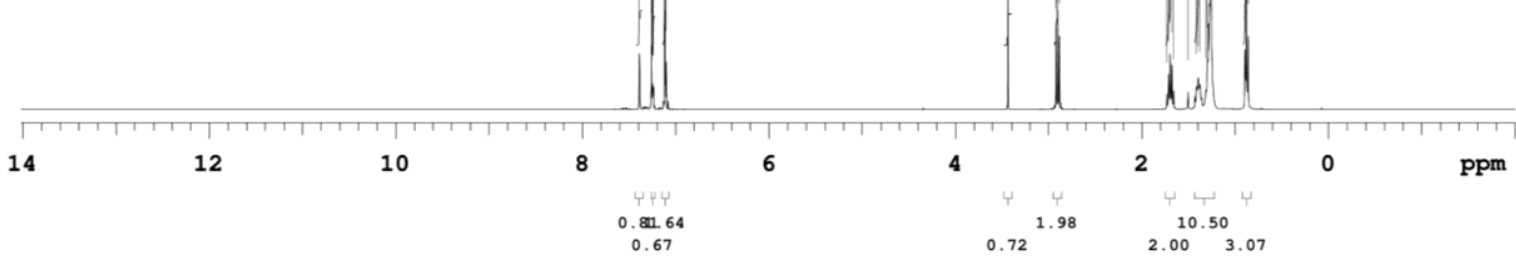


${ }^{13}$ C NMR Spectrum of 3-(Octylseleno)thiophenol

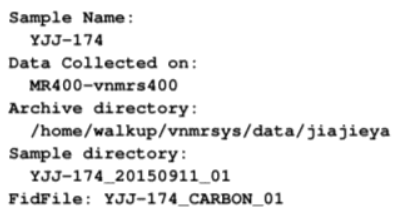<smiles>O=S(=O)(O[Na])c1cccc(S)c1</smiles>

Pulse Sequence: CARBON (s2pul

Solvent: cdc13

Data collected on: Sep 112015

Temp. $25.0 \mathrm{C} / 298.1 \mathrm{x}$

Sample \#6, Operator: jiajieya

Relax. delay $1.000 \mathrm{sec}$

Pulse 45.0 degrees

Acq. time $1.311 \mathrm{sec}$

256 repetitions

OBSERVE C13, 100,5727681 MHE

DECOUPLE H1, 399.9729205 MHE

Power $35 \mathrm{~dB}$

cont inuousiy on

WALTZ-16 modulate

DATA PROCESSING

Line broadening $0.5 \mathrm{~Hz}$

FT size 65536

Total time $9 \min 52 \mathrm{sec}$

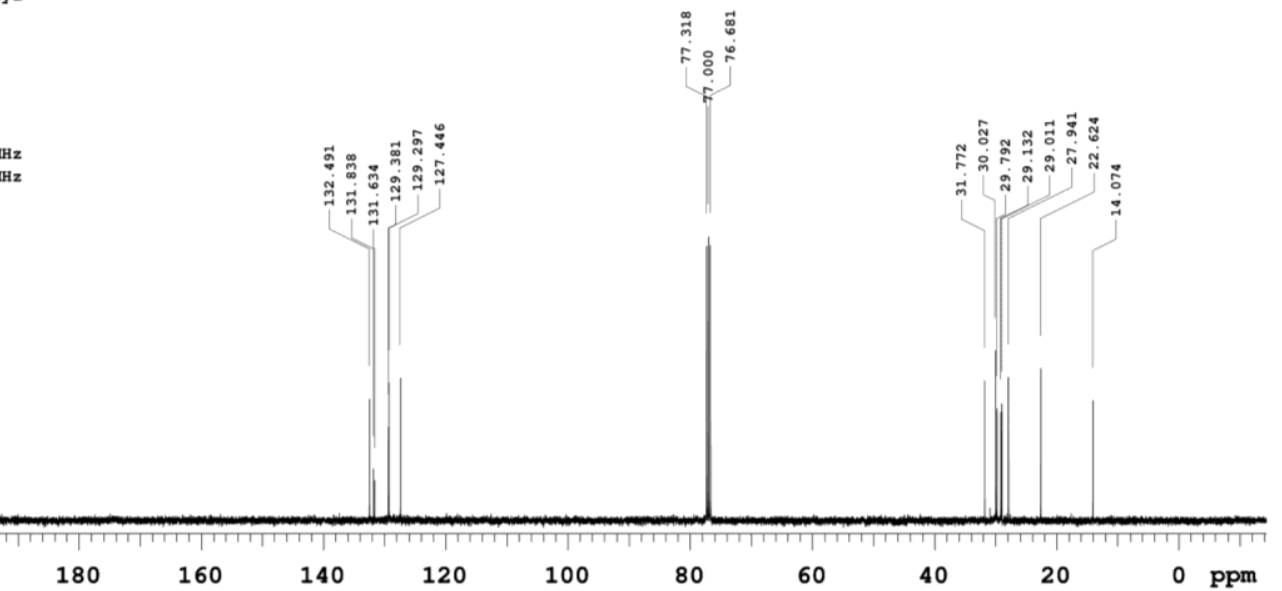

${ }^{77}$ Se NMR Spectrum of 3-(Octylseleno)thiophenol

Sample Name :

Data Collected on:

MR400-vnmrs 400

Archive directory

/home/walkup/vnmrsys/data/jiajieya

Sample directory:

YJJ-174_20150911_01

Pulse Sequence: SELENIUM77 (s2pul)

Solvent: cdc13

Data collected on: Sep 112015

Temp. $25.0 \mathrm{C} / 298.1$

Sample $\#$, Operator: jiajieya

Relax. delay $1.000 \mathrm{sec}$

Pulse 45.0 degrees

Acq. time 1.101 se

128 repetitions

OBSERVE Se77, 76.2805061 MHz

DECOUPLE H1, 399.9729205 MH

Power $35 \mathrm{~dB}$

on during acquisition

off during delay

WALTZ-16 modulat

DATA PROCESSING

Line broadening $0.5 \mathrm{~Hz}$

Total time 4 min $29 \mathrm{sec}$

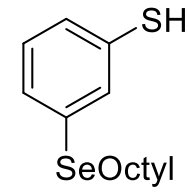

Agilent Technologies

$\stackrel{7}{\pi}$ 
${ }^{1} \mathrm{H}$ NMR Spectrum of Compound $\mathbf{9 b}$

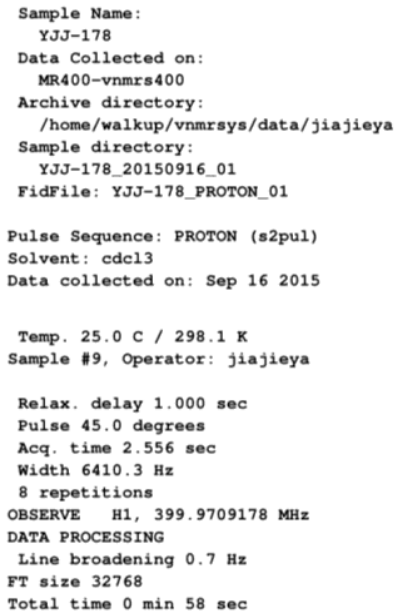<smiles>O=S(=O)(O[Na])c1cccc(SSc2cccc([SeH](=O)(O)OCc3ccccc3)c2)c1</smiles>

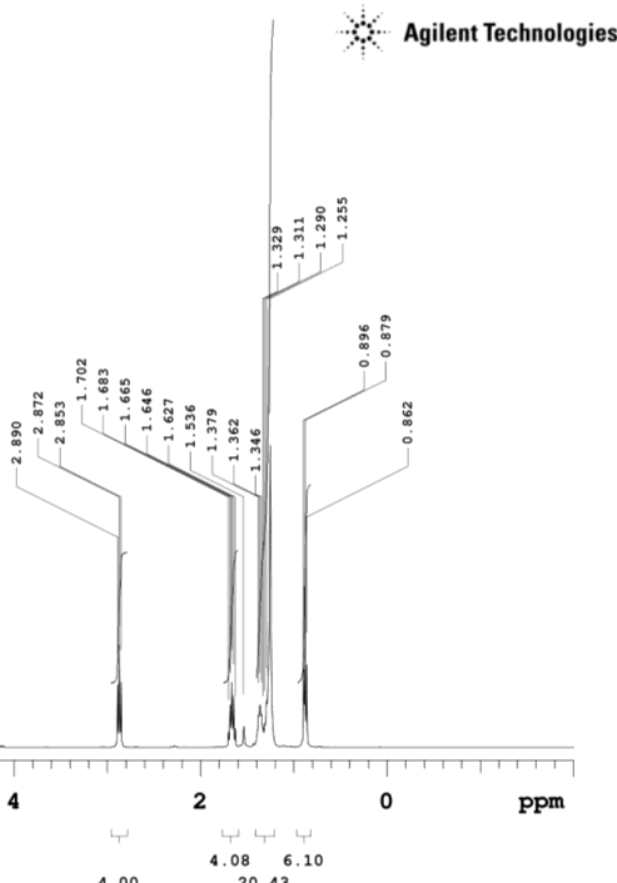

${ }^{13} \mathrm{C}$ NMR Spectrum of Compound $\mathbf{9 b}$

Sample Name:
YJJ-178

Data Collected on

Data Collected on:

MR400-vnmrs400

/home/walkup/vnrsys/data/jiajieya

Sample directory:

YJJ-178 20150916

FidFile: YJJ-178_CARBON 01

Pulse Sequence: CARBON (s2pul)

Solvent: cdc13

Data collected on: Sep 162015

Temp. $25.0 \mathrm{C} / 298.1 \mathrm{~K}$

Sample \#11, Operator: jiajieya

Relax. delay $1.000 \mathrm{sec}$

Pulse 45.0 degrees

Acq. time $1.311 \mathrm{sec}$

width $25000.0 \mathrm{~Hz}$

320 repetitions

OBSERVE C13, 100.5727682 MH

DECOUPLE H1, 399.9729205 MHZ

Power $35 \mathrm{~dB}$

continuously on

WALTZ-16 modulate

DATA PROCESSING

Line broadening $0.5 \mathrm{~Hz}$

FT size 65536

Total time $12 \mathrm{~min}$<smiles>O=S(=O)(O[Na])c1cccc(SSc2cccc([SH](=O)(O)O[Na])c2)c1</smiles>

Agilent Technologies 
${ }^{77}$ Se NMR Spectrum of Compound $\mathbf{9 b}$
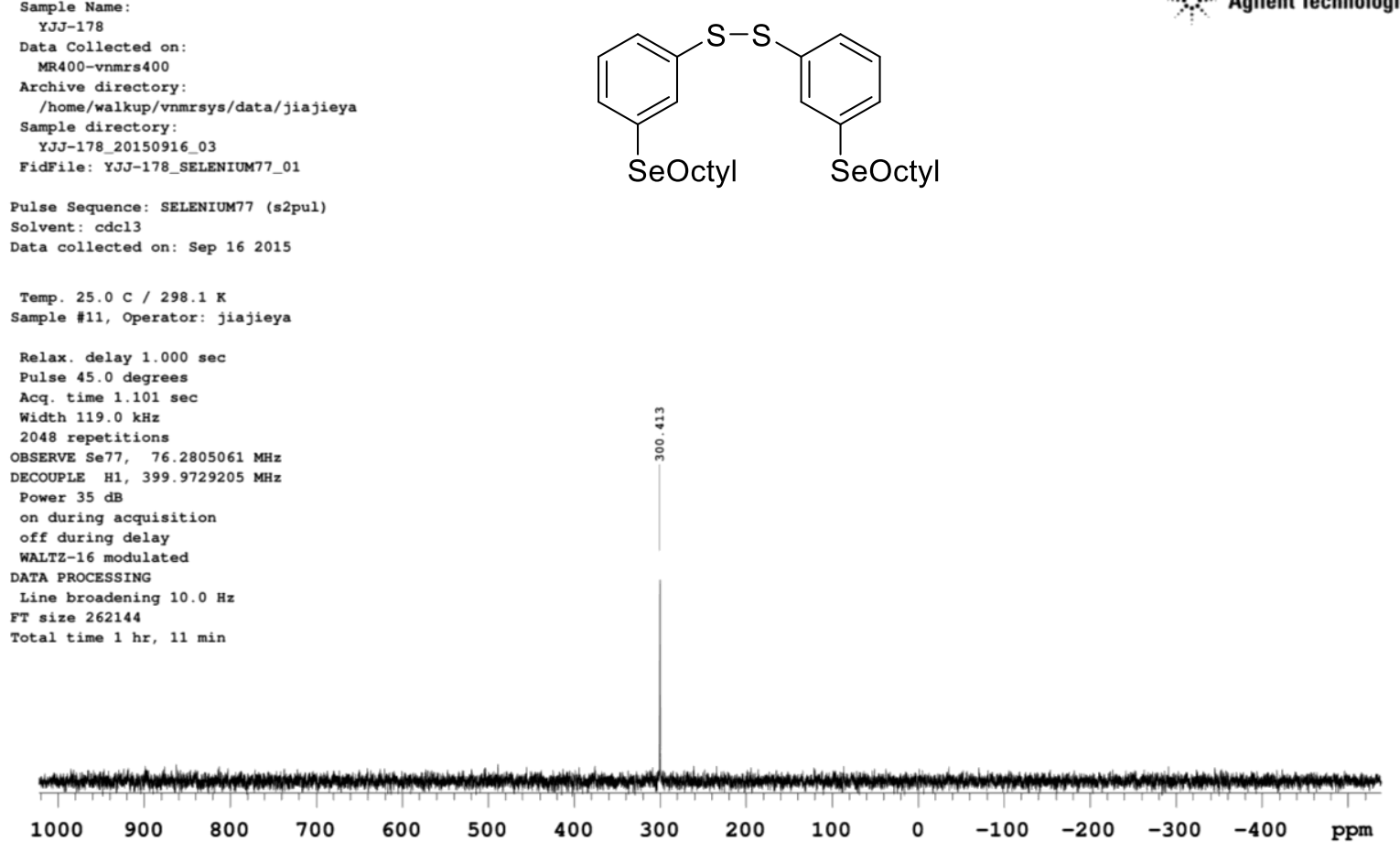

$\begin{array}{lllllllllllllllll}1000 & 900 & 800 & 700 & 600 & 500 & 400 & 300 & 200 & 100 & 0 & -100 & -200 & -300 & -400 & \text { ppm }\end{array}$

${ }^{1}$ H NMR Spectrum of Compound 11

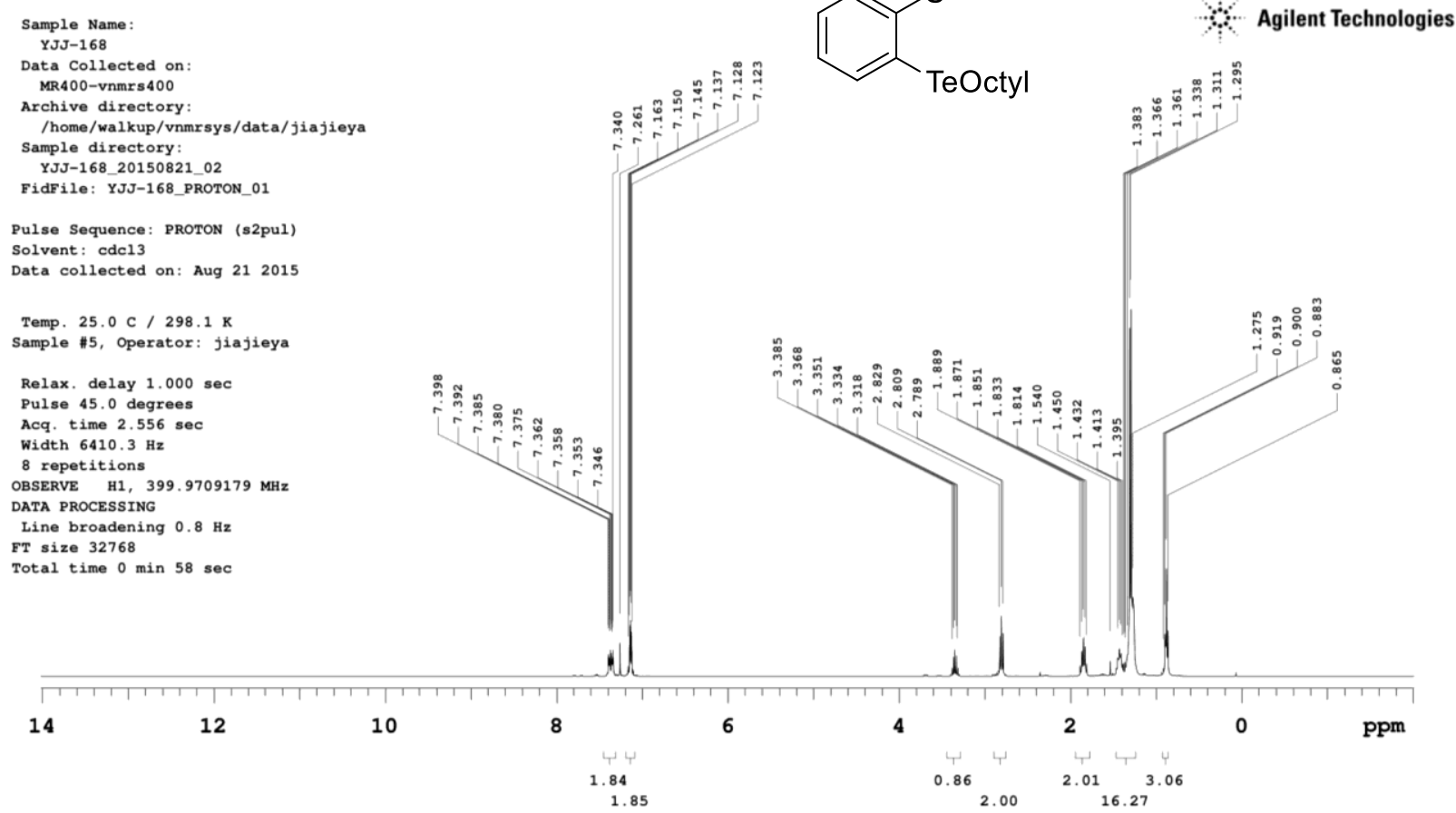


${ }^{13} \mathrm{C}$ NMR Spectrum of Compound $\mathbf{1 1}$

Sample Name :
YJJ-168

Data Collected on:

archive directory

jos/data/jiajieya

YJJ-168 201508:

FidFile: YJJ-168_CARBON_01

Pulse Sequence: CARBON (s2pul)

Solvent: cdc13

Data collected on: Aug 212015

Temp. $25.0 \mathrm{C} / 298.1 \mathrm{~K}$

Sample \#5, Operator: jiajieya

Relax. delay $1.000 \mathrm{sec}$

Pulse 45.0 degrees

Acq. time 1.311 sec

320 repetitions

OBSERVE C13, 100.5727682 MHz

DEC

Power $35 \mathrm{~dB}$

continuously of

WALTZ-16 modulat

DATA PROCESSING

Line broadening $0.5 \mathrm{~Hz}$

FT size 65536

Total time $12 \mathrm{mi}$
MR400-vnmrs400

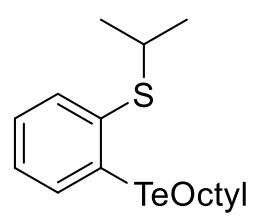

Agilent Technologies$$
\text { TeOcty }
$$

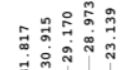

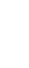$$
\text { - }
$$

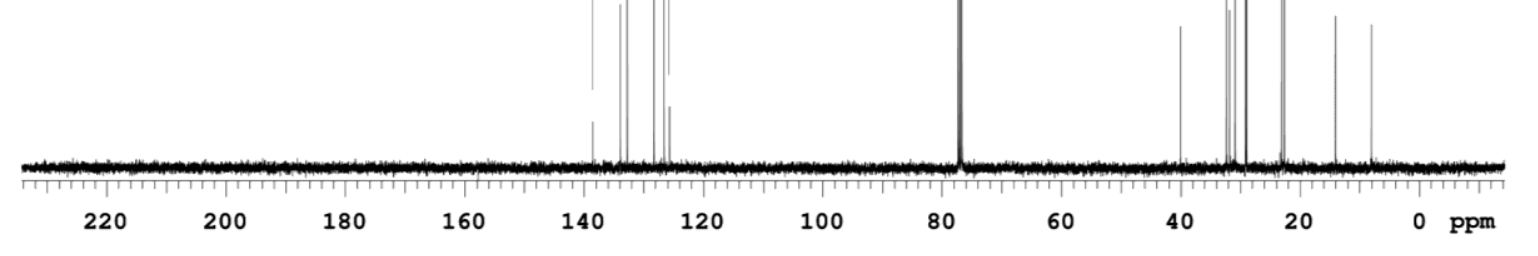

${ }^{125}$ Te NMR Spectrum of Compound 11

\section{Sample Name:}

YJJ-168
Data Collected

Data Collected on :
MR400-vnmrs400

MR400-vnmrs 400
Archive directory

/home/walkup/vnmrsys/data/jiajieya

Sample directory:

YJJ-168_20150821_02
FidFile: YJJ-168_TELLURIUM_01

Pulse Sequence: TELLURIUM (s2pu1)

Solvent: cdc13
Data collected on: Aug 212015

Temp. $25.0 \mathrm{C} / 298.1 \mathrm{~K}$

Sample \#5, Operator: jiajieya

Relax. delay $1.000 \mathrm{sec}$

Pulse 61.7 degrees

Acq. time $0.600 \mathrm{sec}$

Width $357.1 \mathrm{kHz}$

256 repetitions

OBSERVE Te125, 126.1846639 MH

DECOUPLE H1, $399.9729205 \mathrm{MHz}$

Power $35 \mathrm{~dB}$

continuously on

WALTZ-16 modulate

DATA PROCESSING

Line broadening $3.0 \mathrm{~Hz}$

FT size 524288

Total time $6 \mathrm{~min} 50 \mathrm{sec}$

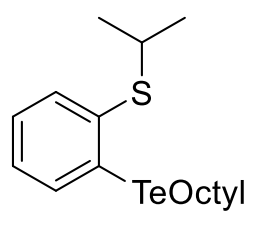

$\stackrel{\infty}{\circ}$

m.

Agilent Technologies

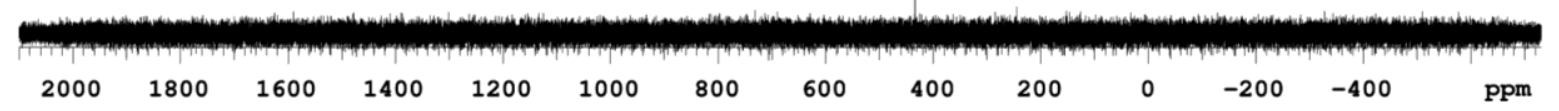


${ }^{1} \mathrm{H}$ NMR Spectrum of Compound $\mathbf{1 3}$

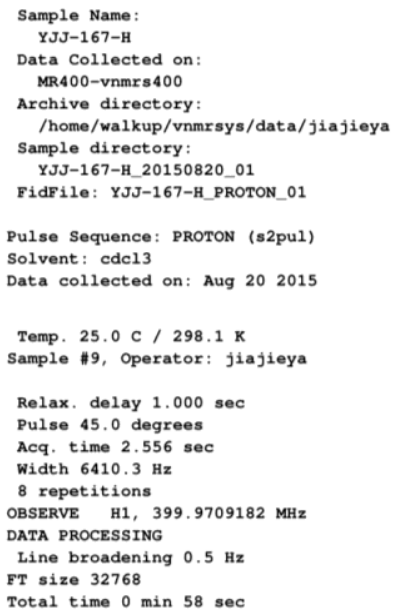<smiles></smiles>

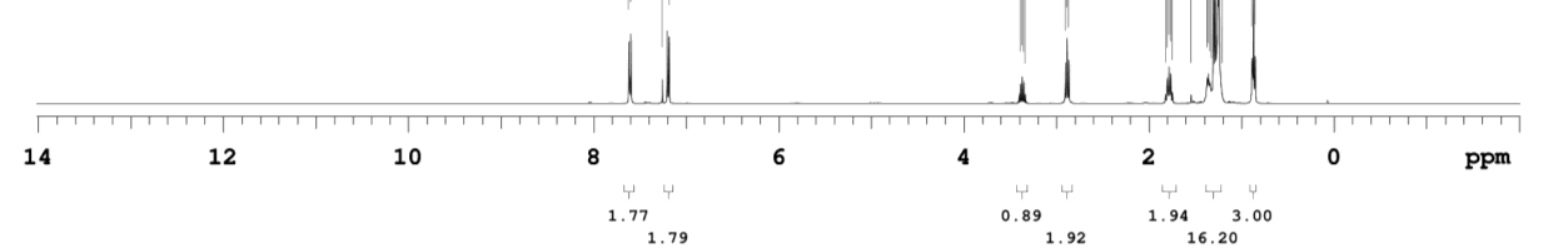

${ }^{13} \mathrm{C}$ NMR Spectrum of Compound $\mathbf{1 3}$

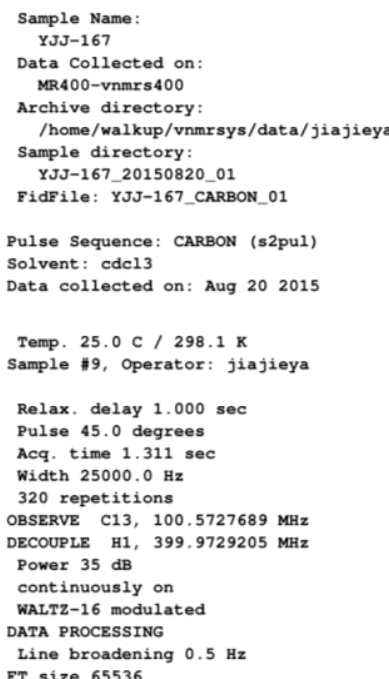<smiles>CC(C)Sc1ccc([Te+2]([O-])O[Na])cc1</smiles>

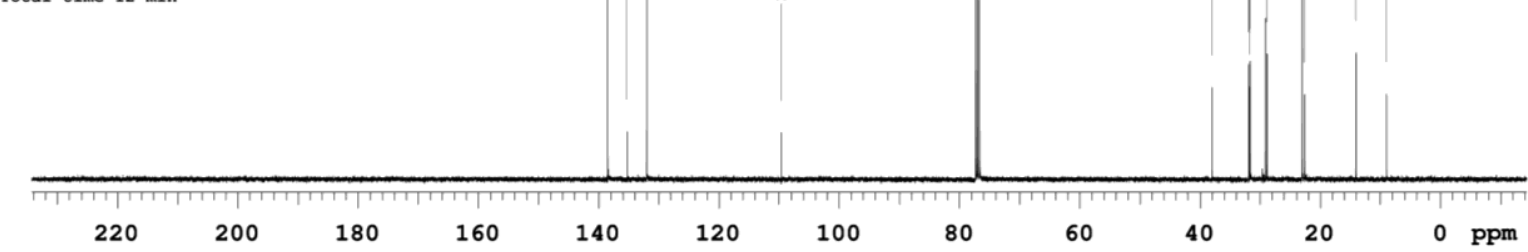


${ }^{125}$ Te NMR Spectrum of Compound $\mathbf{1 3}$

\section{Sample Name:}

YJJ-167

Data collected on:

Archive directory

Archive directory:

/home/walkup/vnmrsys/data/jiajieya

YJJ-167 20150820 01

FidFile: YJJ-167_TELLURIUM_01

Pulse Sequence: TELLURIUM (s2pul)

Solvent: cdc13

Data collected on: Aug 202015

Temp. $25.0 \mathrm{C} / 298.1$

Sample \#9, Operator: jiajieya

Relax. delay $1.000 \mathrm{sec}$

Pulse 61.7 degrees

Acq. time 0.600 soc

Width $357.1 \mathrm{kHz}$

OBSERVE Te125, 126.1846639 MHZ

DSCRve Te125, 126.1046639 $\mathrm{Mrz}$

Power 35 dib

DarT2-16 modulated

Davis 16 modulated

DATA PROCEssiNG

Total time 6 min $50 \mathrm{sec}$

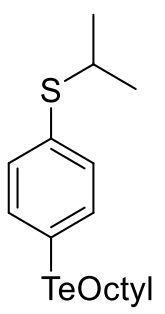

Agilent Technologies

ڤ్

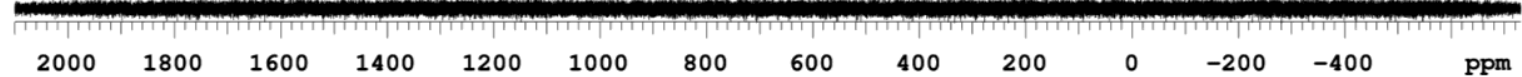

